\title{
CONSIDÉRATIONS SUR LA PROROGATION VOLONTAIRE DE COMPÉTENCE EN MATIÈRE DE DIVORCE ET DE RESPONSABILITÉ PARENTALE
}

\author{
DOI:10.24193/SUBBiur.62(2017).4.3 \\ Published Online: 2017-12-20 \\ Published Print: 2017-12-30
}

\begin{abstract}
Alina OPREA*
Abstract. As a reaction to the complexity created by the diversification and the specialization of the norms governing the international disputes in family matters, the party autonomy is increasingly gaining ground as an effective tool for addressing the problems in this field. In the context of the discussions related with the Brussels II bis regulation's reform, our study is focused on the corresponding position of the European legislator in the field of jurisdiction for international matrimonial and parental responsibility disputes; illustrating some of the existing difficulties and their possible solutions, it also insists on the benefits that an the extension of the party autonomy would have in terms of reinforcement of the legal security, certainty and predictability for those involved in such disputes.
\end{abstract}

Keywords: party autonomy, voluntary prorogation of international jurisdiction, matrimonial disputes, parental responsibility disputes, best interest of the child.

Résumé. Face à la complexité générée par la diversification et la spécialisation des règles applicables au contentieux familial dans les disputes internationales, l'autonomie de la volonté gagne un rôle de plus en plus important comme instrument efficace pour la résolution des problèmes en la matière. Dans le contexte des discussions liées à une éventuelle réforme du règlement 2201/2003, l'étude est focalisée sur les solutions retenues à son égard par le législateur européen pour les litiges internationaux de divorce, séparation de corps ou annulation du mariage, respectivement ceux de responsabilité parentale. Tout en illustrant certaines des difficultés existantes et les solutions possibles, on insistera sur les avantages d'une extension du rôle de l'autonomie de la volonté.

Mots-clés : autonomie de la volonté ; prorogation volontaire de la compétence internationale; contentieux matrimonial ; responsabilité parentale ; intérêt supérieur de l'enfant. 
1. La croissance des flux migratoires met son empreinte sur les sociétés des États européens, qui deviennent de plus en plus internationalisées; les rapports de droit privé présentant des liens avec plusieurs systèmes de droit sont eux aussi une réalité de plus en fréquente. Vu que pour les disputes en matière familiale, sur le fond des divergences importantes entre valeurs et cultures, les réponses offertes par les législations étatiques contrastent significativement en dépit de la similitude des questions soulevées, le traitement juridique qui leur est réservé connaît une complexité accrue et la mission des normes de droit international privé, destinées à gérer ce pluralisme, n'est pas du tout simple. Certes, au niveau de l'Union européenne ont été adoptés des règlements dont le rôle est celui de résoudre de manière uniforme les problèmes concernant la compétence juridictionnelle et la désignation de la loi applicable, et de faciliter la circulation des jugements ${ }^{1}$. Toutefois, ceux-ci n'éliminent que très partiellement la complexité existante, la diversification et la spécialisation des solutions étant une caractéristique dominante y compris pour ce qui est de leur contenu : d'un côté, les textes en discussion consacrent des ensembles doubles (et même parfois triples) de règles, qui se distinguent par leur objet - la compétence juridictionnelle, la loi applicable et la circulation des jugements ; d'un autre côté, ceux-ci instituent des solutions particularisées et différenciées par catégories de rapports juridiques - divorce, responsabilité parentale, régimes matrimoniaux, obligations alimentaires, successions -, ainsi que l'uniformité et la simplification apportées ne sont pas nécessairement évidentes.

Tel est le cas aussi pour le domaine qui fait l'objet de la présente étude : le contentieux matrimonial et de la responsabilité parentale. Pour ce qui est de la compétence juridictionnelle et la reconnaissance et l'exécution des jugements, le règlement européen $2201 / 2003$, qui succède au règlement $1347 / 2000^{2}$, apporte une série de normes uniformes, avec des différenciations importantes toutefois, qui reflètent les particularités de la matière. La dissociation des compétences en fonction de la nature du contentieux (contentieux matrimonial ou responsabilité parentale) a été considérée nécessaire afin de garantir l'existence des critères de compétences qui permettent la saisine de la juridiction la plus proche de la situation litigieuse. Pour chaque catégorie, les critères de compétence ont été multipliés afin de répondre mieux tant aux intérêts des personnes impliquées, qu'aux objectifs spécifiques poursuivis par le législateur européen (spécialement celui de la libre circulation des personnes).

En ce qui concerne la loi applicable, la situation est plus nuancée : le règlement 1259/2010 met en application une forme de coopération consolidée seulement dans le domaine de la loi applicable au divorce et à la séparation de corps ${ }^{3}$. Pour le mariage et son annulation, les règles de droit international privé de chaque État membre gardent leur place ${ }^{4}$; pour ce qui est de l'autorité parentale aussi, la considération des règles nationales de conflit est nécessaire ${ }^{5}$.

2. En ce contexte, l'autonomie de la volonté gagne de plus en terrain comme un instrument efficace de résolution des problèmes soulevés par l'internationalisation des litiges et la complexité résultant de la diversité des règles ${ }^{6}$. Même si cette technique a été traditionnellement réservée à la sphère économique (contrats/obligations extracontractuelles), 
le cadre initial a été dépassé, elle étant plus fréquemment utilisée en matière familiale aussi, suite à un trend inspiré par des conceptions philosophiques qui mettent l'accent sur la valorisation de l'individu' ${ }^{7}$. Les premières extensions ont été opérées dans le domaine des relations de famille ayant un contenu patrimonial (obligations alimentaires, successions, régimes matrimoniaux $)^{8}$, des évolutions importantes étant aussi enregistrées ensuite pour des questions ayant un aspect personnel prédominant, telles le divorce ou la responsabilité parentale.

La croissance du rôle de l'autonomie de la volonté en matière familiale est soutenue non seulement par l'importance des principes d'égalité ou non-discrimination, de la liberté personnelle ou celui du respect de l'identité culturelle des personnes, mais aussi par des raisons tenant aux objectifs du droit international privé et du droit de l'Union européenne. Ainsi, l'autonomie de la volonté introduit un niveau élevé de flexibilité dans le fonctionnement des règles ; elle soutient les intérêts des parties, contribue à assurer la certitude et la sécurité juridique, apportant de la stabilité et de la prédictibilité pour les intéressés, dans un contexte transfrontière qui génère des problèmes délicats. Elle permet un certain niveau d'unité juridique et simplifie le traitement des disputes. Les solutions volontaristes sont de nature à soutenir la mobilité transfrontalière des personnes et, avec cela, les objectifs de la réalisation du marché intérieur ; de même, elles encouragent les parties à rechercher le consensualisme aussi pour leurs problèmes au fond.

Ces avantages incontestables peuvent être exploités aussi par le droit procédural en matière familiale 9 . Le législateur européen a essayé d'en tenir compte, d'autant plus que l'argument du bouleversement de la diversité des systèmes juridiques nationaux et des spécificités des législations étatiques en matière familiale ne joue pas un rôle tellement important au niveau de la compétence juridictionnelle ${ }^{10}$. Dans les litiges liés à la famille (divorce, régimes matrimoniaux, responsabilité parentale, obligations alimentaires), I'autonomie de la volonté facilite une gestion unitaire des problèmes, par la concentration du contentieux devant une seule juridiction, au-delà de la dispersion entraînée par les critères objectifs de compétence, pas toujours corrélés entre eux, apportant ainsi une plus de légitimité aux yeux du public pour les solutions retenues.

La volonté de soutenir les spécificités nationales et l'impérativité traditionnelle des normes en la matière ont imposé toutefois une dose de timidité importante des solutions ; le droit de la famille est imprégné par des conceptions éthiques et morales particulières, propres à chaque État, qui s'articulent plus difficilement avec l'autonomie de la volonté ; celles-ci impliquent des droits et des obligations à l'égard desquels n'existe pas, normalement, la libre disponibilité ${ }^{11}$. La sévérité en la matière, traduite par une limitation de l'autonomie de la volonté, est généralement considérée nécessaire pour ménager des éventuelles craintes de fraude ou d'évasion des normes impératives, pour pouvoir garantir la protection adéquate des standards et maintenir la cohérence des solutions et, également, pour protéger adéquatement les intérêts des personnes vulnérables. 
3. Dans le contexte des discussions liées à la réforme du règlement $2201 / 2003$, pierre angulaire du droit international privé européen en matière familiale, la présente étude est consacrée à l'analyse du lieu et du rôle réservé actuellement par le législateur européen à l'autonomie de la volonté en matière de compétence juridictionnelle pour les litiges de divorce ou annulation du mariage (I), respectivement de responsabilité parentale (II) ${ }^{12}$; l'examen des solutions retenues sera l'occasion d'insister sur les avantages d'un plus d'autonomie en ce domaine et, également, d'illustrer certaines des difficultés existantes et leurs réponses possibles.

\section{La prorogation volontaire de compétence dans les litiges internationaux de divorce ou annulation du mariage}

4. Des critères objectifs de compétence. La réglementation européenne en matière de compétence juridictionnelle pour les litiges de divorce, séparation de corps ou annulation du mariage poursuit des intérêts variés, parmi lesquels figurent indiscutablement la garantie de l'accès égal à la justice et la prévisibilité juridique ${ }^{13}$. Même si l'autonomie de la volonté est de nature à les soutenir, dans le règlement 2201/2003, qui se substitue aux normes nationales de procédure civile ${ }^{14}$, le législateur européen a préféré édicter des critères objectifs de compétence ${ }^{15}$ : la norme générale de l'article 3 prévoit sept critères de compétence alternatifs et concurrents, fondés sur le principe de proximité ; cette multiplication, soutenue par le caractère alternatif des compétences, confère au demandeur une marge de discrétion importante, qui encourage la saisine rapide des instances (rush to the courts) et le phénomène de forum shopping ${ }^{16}$.

L'admission indirecte et limitée de l'autonomie de la volonté. Le jeu de l'autonomie de la volonté est admis seulement de façon indirecte, dans une forme limitée : l'article 3.1, $4^{\mathrm{e}}$ précise que, de commun accord, les époux peuvent introduire leur demande devant les juridictions de l'État membre de la résidence habituelle de chacun d'entre eux. La solution représente une forme extrêmement timide et incomplète d'admission de l'autonomie de la volonté en matière de divorce international, séparation de corps ou annulation du mariage.

Deux conditions cumulatives résultent à la lecture du texte. Tout d'abord, la juridiction choisie doit nécessairement appartenir à l'État de la résidence habituelle de l'un des époux ; peu importe la durée de la résidence (de l'un ou des deux époux) dans cet État avant l'introduction de la demande ou la possession ou non de la citoyenneté de l'État en cause. Le critère de la résidence habituelle doit être interprété de manière autonome, selon les indications tracées par la jurisprudence de la Cour de justice ${ }^{17}$; celle-ci a insisté, dans les affaires avec mineurs, sur l'existence d'un certain niveau d'intégration de la personne dans le milieu de l'État et a offert un éventail des critères qui doivent être pris en compte par les juridictions, dans le contexte de l'analyse de toutes les circonstances pertinentes de l'affaire ${ }^{18}$. 
Tel qu'il est formulé, le texte légal ne permet pas l'option tacite, ni l'option en faveur d'une juridiction autre que celle de la résidence habituelle de l'un des époux. Spécialement, n'est pas permise l'option en faveur des instances de l'État de nationalité de l'un d'entre eux, bien qu'un lien suffisant puisse être considéré comme existent même dans ces cas. La réglementation de cette autonomie de la volonté minimale est par ailleurs déficitaire : ne sont pas établies les conditions de validité (formelle ou substantielle) que l'accord devrait remplir ; pour cela il devra être tenu compte du droit interne de la juridiction saisie (lex fori), avec une éventuelle exigence autonome relative à l'existence d'un consentement informé.

À travers cette limitation, le législateur essaye d'assurer une connexion entre la situation des parties (ou du moins de l'une d'entre elles) et l'État de la juridiction choisie. Si la résidence habituelle des époux est dans un même État, le lien entre le litige et le for correspondant est suffisamment fort : les instances de l'État de la localisation du centre de la vie familiale du couple vont intervenir pour trancher les problèmes liés à rupture du lien matrimonial. Lorsque les résidences des époux sont localisées dans des États différents, l'admission de l'autonomie de la volonté soutient la prévisibilité et permet des économies de temps - s'il obtient l'accord de l'autre, l'époux voulant voir le litige tranché dans l'État de sa résidence habituelle ne devra pas attendre six mois ou une année (selon qu'il possède ou non également la citoyenneté de cet État), pour y introduire sa demande de divorce, séparation de corps ou annulation du mariage. La solution est admissible aussi lorsqu'un des époux n'a plus sa résidence habituelle dans un État membre de l'Union européenne, ce qui révèle son utilité ; toutefois, étant donné que l'autonomie de la volonté est limitée, la règle est inopérante si aucun des époux, tous les deux citoyens des États membres, n'a pas (ou plus) sa résidence habituelle sur le territoire d'un État membre ${ }^{19}$.

Ensuite, la deuxième condition prévue est celle d'une demande commune de divorce, séparation de corps ou annulation du mariage $\mathrm{e}^{20}$; avec cette exigence est présupposée la volonté des époux de prorogation de la compétence de l'instance saisie. Le renvoi à la demande commune pourrait être vu aussi comme un moyen d'assurer l'acceptation expresse de la compétence; la prorogation tacite (dans laquelle un des époux s'adresse à une juridiction, incompétente selon les critères du règlement, et le défendeur se présente devant celle-ci et plaide directement l'affaire sur le fond, sans contester la compétence) n'est pas admissible dans le contexte des règles établies par l'article 3 du règlement ${ }^{21}$.

Le fait que le législateur parle de " demande " pourrait être interprété dans le sens de l'admission de l'autonomie de la volonté seulement dans l'hypothèse de l'existence effective d'un litige et pas comme une technique de prévision de la compétence pour ce qui est des éventuels litiges futurs ; ainsi, les conventions de choix de la juridiction compétente incluses dans des arrangements prémaritaux ou conclues pendant le mariage seraient probablement inefficaces (et ne seraient pas visées par les dispositions de l'article 3.1, $4^{\mathrm{e}}$ du règlement). Ainsi, la référence à la demande commune fait qu'un éventuel accord sur la juridiction compétente soit insuffisamment protégé ; si la juridiction choisie n'a pas été saisie, tandis que l'une des parties s'adresse à une autre juridiction, compétente selon l'article 3 du règlement, cette dernière aura priorité. 
5. Discussions liées à une possible reforme. En 2006, la Commission européenne a avancé une proposition de réforme du règlement $2201 / 2003^{22}$ : ouverte aux objectifs de garantie de la sécurité juridique, de la prévisibilité, de la flexibilité, de facilitation de l'accès à la justice et de diminution de la course vers les tribunaux, elle proposait un nouvel article 3 bis $^{23}$, dédié au choix par les parties de la juridiction compétente en matière de divorce et séparation de $\operatorname{corps}^{24}$. Celui-ci représentait une première ouverture véritable pour l'admission de l'autonomie de la volonté en la matière ${ }^{25}$.

La solution était suffisamment flexible et encourageait la coopération entre les époux ; pour soutenir la prévisibilité et pour garantir l'existence d'un consentement réel et effectif, seulement l'option expresse était admise, et pas aussi la prorogation tacite de la compétence $^{26}$. Le texte incluait toujours des limitations, destinées à éviter la création des paradis du divorce : le jeu de l'autonomie de la volonté était permis seulement en faveur des juridictions des États avec lesquels les époux présentaient des liens étroits. Ainsi, plus concrètement, les critères de l'article 3 du règlement étant présumés exprimer des tels liens étroits, il était permis aux époux d'opter pour une des instances qui seraient compétentes sur la base de ce texte légal ; tenant compte de l'effet (subsidiaire) dérogatoire d'une telle option, la solution était de nature de renforcer la sécurité juridique et la certitude pour les parties. Si l'accord en faveur des instances de l'État de la résidence habituelle de chacun des époux était une alternative (la seule) disponible aussi dans le cadre de l'article 3 du règlement 2201/2003, dans le cas de l'introduction d'une demande commune, la proposition permettait supplémentairement l'option en faveur des instances de l'État de nationalité de chacun des époux ; celle-ci était justifiée par la facilitation de l'accès à la justice et avait comme fondement le lien culturel existant entre les époux et l'État respectif. Enfin, parmi les alternatives apparaissait également la possibilité de désigner les instances de l'État de la dernière résidence commune des époux (si l'habitation là-bas a duré au moins trois années), option avec des effets favorables spécialement pour les citoyens des États tiers n'habitant plus dans l'Union.

À côté de cette ouverture bienvenue vers un plus de liberté en la matière, la proposition de 2006 méritait à être appréciée aussi du point de vue des précisions apportées sur la forme de l'accord, qui devrait être un écrit signé par les deux parties au plus tard au moment de la saisine de la juridiction. Elle soulevait toutefois quelques inconvénients. Ainsi, bien que la signature fonctionne comme une garantie du consentement effectif, sa simple présence n'élimine pas les problèmes liés à l'expression d'un consentement informé, qui assure un minimum de protection aux personnes vulnérables; de ce point de vue, la proposition apparaissait déficitaire. Le même caractère déficitaire était mis en évidence par un autre aspect : en n'imposant aucune limitation relative à l'efficacité temporale de l'accord, la solution proposée ouvrait les portes aux conventions attributives de juridiction contenues dans des pactes prénuptiaux ou conclus à tout moment pendant le mariage. Or, tant du point de vue de l'indisponibilité des droits substantiels en discussion, que de celle de la protection de l'époux vulnérable par la limitation sévère de son droit d'accès effectif à un juge (en contraste par ailleurs avec l'ouverture montrée par le législateur dans l'article 3), l'opportunité d'une si ample liberté pouvait soulever des doutes. 
6. La proposition de réforme de 2006 a été temporairement abandonnée, le législateur européen concentrant ses efforts dans le domaine des conflits de lois ${ }^{27}$. Toutefois, en 2014, suite au Rapport de la Commission relatif à l'application du règlement $2201 / 2003^{28}$ et dans l'idée de continuer le développement de l'espace européen de la justice et des droits fondamentaux, les initiatives de modification sont réapparues sur l'agenda européen. Même si l'étude effectuée par Deloitte ${ }^{29}$ a mis en évidence le fait que les règles existantes ne promeuvent pas adéquatement le consensus entre les époux, en les privant ainsi de l'accès aux instances qui leur sont les plus convenables ${ }^{30}$ (effet soutenu également par les interprétations non-uniformes du concept résidence habituelle), et une proportion majoritaire des répondants consultés en vue de la réalisation de l'étude a soutenu l'opportunité de l'admission de la liberté des époux de se mettre d'accord sur la juridiction compétente ${ }^{31}$, la proposition de la Commission de réforme du règlement 2201/2003 du juin 2016 ne contenait pas des règles en ce sens ${ }^{32}$.

Justifiée probablement par la crainte que l'autonomie de la volonté pourrait être utilisée pour contourner la compétence des systèmes juridiques qui imposent des contraintes plus élevées en la matière afin de favoriser la stabilité du mariage et les obligations familiales au détriment de la volonté privée, la solution peut néanmoins être critiquée. Premièrement, en procédant de cette façon, le législateur européen n'assure pas la cohérence nécessaire dans la réglementation : même si l'autonomie de la volonté est admise en matière de divorce international pour ce qui est de la résolution des conflits de ${ } \mathrm{ois}^{33}$, les choses sont différentes en matière de compétence juridictionnelle; de même, bien que dans d'autres matières liées à la sphère familiale - telles la responsabilité parentale, les obligations alimentaires, les régimes matrimoniaux, les successions - la prorogation volontaire de la compétence est admise, pour ce qui est de la dissolution du lien matrimonial le législateur européen poursuit la solution traditionnelle, restrictive. Également, parce que la solution en matière de compétence juridictionnelle contraste avec celle retenue pour ce qui est de la loi applicable au divorce, une autre critique qui pourrait être adressée au législateur européen est celle de ne pas soutenir adéquatement la coïncidence entre la compétence législative et celle juridictionnelle, même si les avantages qu'elle présente en termes de réduction des coûts et d'un plus de célérité dans les procédures qu'elles militent pour une solution en sens contraire ; supplémentairement, étant donné ce domaine particulier dans lequel les réglementations substantielles et celles procédurales sont étroitement imbriquées, une telle coïncidence aurait été d'autant plus nécessaire. Par ailleurs, l'impossibilité de choisir la juridiction compétente peut priver parfois d'effet des conventions d'élection de la loi applicable au divorce, avec toute l'incertitude juridique afférente : ainsi, si l'instance désignée sur la base des critères objectifs de compétence appartient à un État dans lequel le règlement 1259/2010 n'est pas obligatoire, État qui n'admet non plus la professio juris en matière de divorce, le choix de la loi applicable par les parties serait inopérant, avec toutes les conséquences négatives du point de vue de l'affectation des leurs expectatives légitimes. Enfin, l'autonomie attendue n'était pas une autonomie ample, selon le modèle consacré en matière civile et commerciale par les articles 25 et 26 du règlement 1215/2012; l'équilibre voulu entre les systèmes plus libéraux et ceux plus traditionnels impose des limitations dont l'utilité est généralement reconnue, et la Commission en aurait pu tenir compte. 
7. Au contraire, à notre avis, pour encourager le consensualisme, la volonté des parties devrait avoir, de lege ferenda, une place plus grande dans le futur règlement de refonte. Le principe de l'autonomie de la volonté, sous la forme de la prorogation volontaire (et même tacite) de la compétence pourrait avoir une importance accrue, grâce à son utilité pratique pour les parties et pour la bonne administration de la justice : corrélé avec le principe favor divortii, il soutiendrait non seulement la flexibilité des solutions, mais également l'accès à la justice pour les parties impliquées ${ }^{34}$. L'autonomie de la volonté favorise les démarches de type consensuel-collaboratif-conciliateur entre les parties et est de nature à diminuer le phénomène de race to the courts, corrélatif du tourisme judiciaire/ forum shopping ${ }^{35}$, ainsi que son admission serait souhaitable.

Toutefois, les limitations sont nécessaires, afin d'assurer un lien suffisant entre la juridiction et le litige, la protection suffisante de l'impérativité étatique dans une matière reconnue comme délicate, et également afin de réduire la possibilité de l'époux qui se trouve dans une position de force d'en profiter de façon injustifiée au détriment de l'autre. Cela pourrait être réalisé, d'un côté, par la limitation des options des parties quant aux instances qui peuvent être choisies, avec l'introduction des exigences de proximité, et d'un autre côté, à travers des règles strictes relatives à la validité formelle des conventions d'electio fori, garantissant que les personnes impliquées sont conscientes des conséquences de leur choix. Les limites relatives au moment du choix (dans le sens de l'acceptation seulement des conventions conclues après l'éclatement de la dispute, après la survenance du problème de la dissolution du lien matrimonial entre les époux) pourraient se relever également opportunes : une telle solution serait cohérente avec la solution générale du règlement, qui multiplie les critères de compétence, pour répondre plus amplement aux besoins et aux attentes des époux, tout en assurant en même temps l'accès effectif aux instances.

\section{La prorogation de la compétence dans les litiges en matière de responsabilité parentale}

8. Contrairement à la solution rigide retenue pour les litiges de divorce, séparation de corps ou annulation du mariage, le législateur européen s'est montré plus ouvert à l'autonomie de la volonté des parties en ce qui concerne les litiges en matière de responsabilité parentale. Résultat des réformes du règlement $1347 / 2000^{36}$, l'article 12 du règlement 2201/2003 est l'expression de cette ouverture. Le texte introduit un for alternatif au for de la résidence habituelle de l'enfant, considéré le mieux placé pour trancher les disputes relatives à la responsabilité parentale ${ }^{37}$, et est inspiré par un double objectif. D'une part, à travers la possibilité de la concentration du contentieux devant une juridiction unique, celle saisie avec l'action de divorce, séparation de corps ou annulation du mariage ou éventuellement avec une autre action ${ }^{38}$, est soutenue la bonne administration de la justice et l'économie procédurale et est introduite un plus de flexibilité dans le fonctionnement du règlement, mieux adaptée au besoin du respect minimal de l'intérêt supérieur de l'enfant. En même temps, bien que l'autonomie de la volonté ait une marge importante d'action, ses 
limitations ne sont non plus absentes, en vue d'assurer tant un lien substantiel entre le mineur et l'État de la juridiction choisie que, également, la priorité du critère de principe de l'article 8 , inspiré par des considérants de proximité ${ }^{39}$ et efficience ${ }^{40}$.

Le législateur européen différencie entre les exigences relatives au domaine matériel de la prorogation et les exigences relatives à la prorogation même et, en ce qui suit, on va s'y arrêter ponctuellement.

\section{Le cadre général pour l’admission de la prorogation}

Le texte de l'article 12 opère une distinction entre les litiges relatifs à la responsabilité parentale concomitants à ceux de divorce, séparation de corps ou annulation du mariage (art. $12.1)^{41}$ et, respectivement, les litiges non corrélés avec ceux de divorce (art. 12.3 et 12.4). Les règles seront appliquées qu'il s'agisse ou non des enfants communs des époux impliqués dans la dispute ${ }^{42}$.

9. Actions corrélées aux actions de divorce, séparation de corps ou annulation du mariage. Un premier cas (limitatif) dans lequel est admis le jeu de l'autonomie de la volonté dans les litiges relatifs à la responsabilité parentale est celui dans lequel entre les parties existe déjà une procédure pendante de divorce, séparation de corps ou annulation du mariage, la prorogation étant possible en faveur des instances compétentes à statuer sur celle-ci, lorsque (au moins) une des parties de cette procédure exerce la responsabilité parentale à l'égard de l'enfant ; cette dernière exigence est de nature à garantir que la prorogation sera décidée en fonction de l'intérêt supérieur de l'enfant, et non seulement en fonction des intérêts de ses parents (et/ou des titulaires de la responsabilité parentale).

Le moment de l'appréciation d'une procédure pendante de divorce, séparation de corps ou annulation du mariage est celui de la saisine de la juridiction avec l'action en responsabilité parentale. La condition de l'existence de cette procédure peut être justifiée par la volonté d'assurer un lien raisonnable entre la situation de l'enfant et celle de ses parents, impliqués dans la procédure de divorce, séparation de corps ou annulation du mariage ; en ce sens, le législateur européen impose que l'instance saisie ait retenu sa compétence sur la base de l'un des critères prévus par l'article 3 du règlement et non pas, éventuellement, sur la base des règles nationales de procédure civile internationale, dans les conditions des articles 6 et $7^{43}$; en ces derniers cas, le lien du litige de divorce, séparation de corps ou annulation du mariage avec l'espace européen n'est pas suffisamment caractérisé et l'extension de la compétence de l'instance saisie avec ce litige aussi aux disputes concernant la responsabilité parentale peut paraître exorbitante ${ }^{44}$.

Étant une procédure dérivée de la procédure de divorce, séparation de corps ou annulation du mariage, la compétence prorogée sur la base de la règle de l'article 12.1 cessera avec celle-ci. Spécialement, selon l'article 12.2, la compétence de la juridiction va cesser lorsque le jugement relatif à l'anéantissement du lien matrimonial acquiert force de chose jugée, lorsqu'un jugement définitif est prononcé dans le litige relatif à la responsabilité 
parentale, en cours lorsque le jugement de divorce, séparation de corps ou annulation est devenu définitif ou lorsque les litiges de divorce, séparation de corps ou annulation du mariage ou responsabilité parentale ont cessé pour quelconque motif (par exemple, parce que les demandes ont été retirées ou se sont périmées ou parce que l'une des parties est décédée $)^{45}$.

Une précision supplémentaire s'impose. La prorogation va opérer normalement lorsque l'enfant a sa résidence habituelle sur le territoire d'un État membre de l'Union européenne. Lorsque l'enfant a sa résidence habituelle dans un État tiers, une distinction doit être opérée. Si cet État tiers n'est pas un État membre de la Convention de La Haye de 1996, l'article 12 du règlement est pleinement applicable et les instances d'un État membre saisies avec l'action de divorce, séparation de corps ou annulation du mariage pourront se prononcer aussi sur les problèmes de responsabilité parentale. Si l'État (tiers) de la résidence habituelle de l'enfant est un État membre de la Convention de La Haye, cette dernière aura priorité au règlement (art. 61) et les instances vont vérifier leur compétence internationale sur la base de la Convention ${ }^{46}$. Son article 10 étant rédigé dans des termes proches à ceux de l'article $12 \mathrm{du}$ règlement, les solutions obtenues concrètement ne vont pas être tellement différentes. Deux différences doivent toutefois être mentionnées. D'abord, selon la Convention de La Haye, la prorogation est admissible seulement si elle est permise aussi par la loi (procédurale) interne de l'État dont les instances sont compétentes à statuer sur le divorce/l'annulation du mariage, tandis que le législateur européen n'a pas imposé dans le règlement 2201/2003 une telle exigence, consacrant directement un critère de compétence juridictionnelle distinct et autonome ${ }^{47}$. Ensuite, l'article 10 de la Convention conditionne l'admission de la prorogation non seulement de l'exercice de l'autorité parentale par l'un des parents, mais également de la localisation de la résidence habituelle de l'un de ceux-ci dans l'État de l'instance dont la compétence va être prorogée ; cette dernière exigence n'apparaît non plus dans l'article 12 du règlement.

10. Actions en responsabilité parentale non corrélées avec des actions de divorce, séparation de corps ou annulation du mariage. Lorsqu'il n'y a pas un litige en cours sur la dissolution du lien matrimonial, la prorogation volontaire de la compétence est toujours possible $^{48}$. La solution, de nature à apporter un plus de souplesse dans le système des règles consacrées en matière de responsabilité parentale, a un double fondement : l'autonomie de la volonté peur jouer seulement si la proximité est également respectée.

Pour que la prorogation soit admissible, une condition spécifique est imposée: l'existence d'un lien substantiel entre l'enfant et l'État membre dont les juridictions sont saisies, cet État n'étant pas celui de la résidence habituelle de l'enfant. Selon le texte légal, ce lien substantiel/étroit peut normalement exister avec l'État membre dont l'enfant est ressortissant ou avec l'État membre de résidence habituelle de l'un des titulaires de la responsabilité parentale. L'énumération de ces critères n'est pas exhaustive ; concrètement seront prises en compte toutes les circonstances de vie du mineur et de ses parents (titulaires de la responsabilité parentale). L'accent devra tomber non sur des aspects juridiques et formels, mais sur l'effectivité des liens entre l'enfant et un certain État, démontrés par des indices multiples, univoques ou concordants; en ce contexte, l'appréciation globale de ces circonstances est 
essentielle, pas toujours un élément de ceux indiqués par le législateur révélant le lien substantiel nécessaire (mais, au contraire, un simple lien formel) ${ }^{49}$. La Cour de justice $n^{\prime}$ a pas offert dans sa jurisprudence des détails significatifs sur ce lien substantiel ${ }^{50}$, qui devrait être déduit de l'évaluation objective de la situation de fait.

11. La formulation littérale de l'article 12.3 est de nature à soulever des discussions quant à la question de savoir si la prorogation peut être envisagée seulement au cas de l'existence d'une autre action pendante entre les parties ou y compris indépendamment de celle-ci ${ }^{51}$. Le guide pratique pour l'application du règlement, élaboré par la Commission, ne prends pas position ${ }^{52}$, mais le problème a fait l'objet d'une question préliminaire adressée à la Cour de justice par la Cour suprême de la République Tchèque, dans l'affaire L. C. $M^{53}$. En pratique, les difficultés étaient occasionnées par le fait que la lecture combinée des alinéas 1 et 3 de l'article 12 permettait deux interprétations opposées : une, plus restrictive, dans le sens que la prorogation devrait opérer seulement en faveur d'une instance saisie avec une procédure de divorce, séparation de corps ou annulation du mariage, dont la compétence a été établie à la base de l'article 7 du règlement ${ }^{54}$, respectivement, une autre plus libérale, inspirée par l'autonomie de la matière « responsabilité parentale » par rapport au contentieux matrimonial dans le règlement, et qui ne conditionnait pas la prorogation de la connexité entre la procédure en matière de responsabilité parentale et une autre procédure (en cours) $)^{55}$.

En suivant une interprétation systémique et téléologique, la Cour arrive à accorder préférence à cette dernière solution : « La prorogation de compétence prévue à l'article 12, paragraphe 3 , du règlement $n^{\circ} 2201 / 2003$ en matière de responsabilité parentale peut être appliquée sans qu'il soit nécessaire que la procédure en cette matière se rattache à une autre procédure déjà pendante devant la juridiction en faveur de laquelle la prorogation de compétence est souhaitée » (par. 45). L'interprétation large est justifiée par la volonté d'assurer l'effectivité de la disposition, elle étant la seule en mesure de garantir le respect des objectifs poursuivis par le règlement. La Cour insiste sur la nécessité de l'interprétation des dispositions du règlement en tenant compte du principe de l'intérêt supérieur de l'enfant et apprécie qu'une éventuelle subordination du jeu de la prorogation volontaire à l'existence d'un litige en cours irait à l'encontre de ce principe, étant donné la limitation importante qu'elle est susceptible d'entraîner en pratique (par. 48). En même temps, elle considère que l'objectif mentionné dans le considérant 5 du préambule du règlement - assurer l'égalité de tous les enfants - serait lui aussi contrarié par une telle exigence, dont le résultat serait l'exclusion de (presque) toute possibilité de prorogation de la compétence pour les litiges relatifs à des enfants dont les parents n'ont jamais été mariés ou sont déjà divorcés, séparés ou dont le mariage a été déjà annulé (par. 51$)^{56}$.

En tenant compte de la position de la Cour de justice dans cet arrêt, la Proposition de la Commission de juin 2016 relative à la réforme du règlement 2201/2003 supprime la mention interprétable " dans d'autres procédures que celles mentionnées au premier alinéa », figurant actuellement à l'article 12.357. Cette solution est de nature à apporter un plus de clarté des solutions dans le futur et contribue à l'application uniforme du texte. 
12. Un autre aspect discuté en rapport avec la règle de l'article 12.3 est celui de savoir si une juridiction dont la compétence a été prorogée suite à l'accord des titulaires de la responsabilité parentale reste compétente aussi pour statuer dans d'autres litiges futurs entre ceux-ci concernant la situation de l'enfant.

Dans l'affaire E c. B, tranchée en $2014^{58}$, la Cour de justice en a offert une réponse négative : "la compétence en matière de responsabilité parentale, prorogée, en vertu de l'article 12, paragraphe 3, du règlement $n^{\circ} 2201 / 2003$, en faveur d'une juridiction d'un État membre saisie d'un commun accord d'une procédure par les titulaires de la responsabilité parentale, disparaît avec le prononcé d'une décision passée en force de chose jugée dans le cadre de cette procédure " (par. 50 et dispositif). Celle-ci s'est fondée sur une interprétation littérale des termes du règlement, soutenue également par des arguments téléologiques. En tenant compte du fait que les mesures prises en matière de responsabilité parentale sont nécessairement modifiables dans le temps, la Cour a commencé son raisonnement par l'analyse des dispositions des articles 8.1 et 12.3 et a apprécié que la position du législateur européen ait été assez claire dans le sens de la vérification de la compétence de l'instance au moment de sa saisine. Le principe traditionnel en la matière - celui de la cessation de la compétence avec le prononcé du jugement (lata sententia judex desinit esse judex) - a été réaffirmé : l'instance saisie doit vérifier et établir sa compétence " dans chaque cas particulier ", " ce qui implique qu'elle n'est pas maintenue au-delà du terme d'une procédure pendante » $(\text { par. 40 })^{59}$. Supplémentairement, ayant comme prémisse la même indépendance des procédures successives, la Cour a pris en considération les termes de l'article 12.3 du règlement, qui prétendent l'acceptation de la prorogation de la compétence " expressément » ou " de toute autre manière non équivoque ", et a refusé d'admettre que l'accord donné pour une procédure puisse opérer pour toutes les procédures éventuelles relatives à l'autorité parentale, qui pourraient survenir pendant la minorité de l'enfant ${ }^{60}$. La position a été soutenue également avec des arguments liés aux objectifs du règlement, en particulier celui du respect de l'intérêt supérieur de l'enfant. Ainsi, même si le for de la résidence habituelle de l'enfant, inspiré par des considérants de proximité, est prévu alternativement avec celui convenu par les parties, les conditions pour l'admissibilité de ce dernier sont assez restrictives, les juges jouissant à cet égard d'une marge suffisante d'appréciation. Or, admettre que la prorogation convenue à un certain moment opère aussi après la finalisation de la procédure spécifique, bien que les circonstances et le milieu de vie de l'enfant se soient modifiés significativement dans le temps, signifie ignorer cet intérêt supérieur.

\section{B. Conditions de la prorogation}

En plus des particularités de chacune des deux hypothèses qui tracent le cadre général de la prorogation, deux exigences communes sont imposées : l'accord des parties et le respect de l'intérêt général de l'enfant. Étant d'application cumulative, le non-respect de l'une d'entre elles fait obstacle au jeu de la prorogation. 


\section{L'accord des parties sur la prorogation de la compétence}

1.1. Une condition essentielle pour l'applicabilité de l'article 12 est celle de l'extériorisation de la volonté des parties, expressément ou de toute autre manière non équivoque, dans le sens de l'acceptation de la compétence des instances saisies. Avec cela, le caractère principal du critère de la résidence habituelle, probablement le plus adéquat critère de compétence juridictionnelle pour les litiges en matière de responsabilité parentale, est maintenu et est assuré une justification adéquate pour la réalisation de la prorogation, la simple concentration du contentieux n'étant pas suffisante. L'autonomie de la volonté justifie cette dérogation à la règle de la résidence habituelle de l'enfant par les avantages qu'elle présente : prévisibilité, flexibilité, adaptation aux besoins concrets des parties.

Le terme " partie » aura un sens particulier. Pour ce qui est de l'art. 12.1, le législateur précise expressément que la prorogation de la compétence de l'instance saisie avec la dispute matrimoniale pourra opérer en cas d'accord en ce sens des époux et des titulaires de la responsabilité parentale ; si ces personnes peuvent coïncider, tel n'est pas toujours le cas, vu l'extension opérée par le règlement Bruxelles II bis à tous les litiges en matière de responsabilité parentale, qu'ils visent ou non les enfants commun d'un couple ou les enfants sur lesquels les parents exercent ou non la responsabilité parentale. Pour ce qui est de l'article 12.3, la prorogation doit être acceptée par les " parties à la procédure "; la notion vise non seulement les titulaires de la responsabilité parentale (parents ou non), mais également toute autre personne ayant un intérêt à agir dans la procédure de responsabilité parentale, telle que définie par les articles 1.2 et 2.7 du règlement, comme par exemple un grand parents, un ancien époux ou partenaire du titulaire de responsabilité parentale ${ }^{61}$.

Parce que l'absence de l'accord fonctionne comme limite principale de la prorogation de compétence et puisque l'article 12 n'est pas applicable seulement aux demandes communes, la clarification de la signification de l'expression "acceptation expresse ou de toute autre manière non équivoque » de la compétence acquiert une importance primordiale ${ }^{62}$. Celle-ci soulève parfois des difficultés ou hésitations en pratique ${ }^{63}$, et la Cour de justice a été appelée déjà en deux occasions à se prononcer sur les exigences afférentes.

1.2. Le premier arrêt relevant est l'arrêt $L c . M$, du 2014, dont les faits ont été présentés plus haut. Dans l'affaire, par sa deuxième question préliminaire, la juridiction de renvoi voulait obtenir des clarifications sur l'interprétation de l'expression "acceptation expresse ou de toute autre manière non équivoque de la compétence ", dans les conditions de l'existence de deux actions pendantes devant la même juridiction, ayant le même objet, la partie qui s'est adressée à l'instance la deuxième, contestant ultérieurement, à l'occasion du premier acte effectué, la compétence de celle-ci dans la procédure initiée antérieurement par l'autre partie, et s'adressant de façon concomitante à une juridiction d'un autre État membre. Le problème n'était pas simple. L'absence de l'accord sur la prorogation est claire lorsque les parties introduisent des procédures distinctes devant des juridictions des États différents ${ }^{64}$. Toutefois, dans l'affaire, la particularité était donnée par le fait que les deux procédures avaient été introduites devant la même juridiction (et, sur la base du droit procédural interne 
elles auraient dû probablement être jointes) et, la formulation de la demande devant une juridiction particulière fait présumer éventuellement l'acceptation (par le demandeur) de la compétence de ladite juridiction.

La Cour suit une position restrictive en la matière. Elle réalise une analyse littérale des articles 12.3 et 16 du règlement et considère que la date à laquelle doit être appréciée l'existence de l'accord sur la compétence est " au plus tard [...] la date à laquelle l'acte introductif d'instance ou un acte équivalent est déposé auprès de la juridiction choisie " (par. 56). Tenant compte du comportement du défendeur dans la première action qui, même s'il a initié sa propre procédure, a contesté très vite la compétence de l'instance, elle décide que : "l'article 12, paragraphe 3, sous b), du règlement $n^{\circ}$ 2201/2003 doit être interprété en ce sens qu'il ne peut pas être considéré que la compétence de la juridiction saisie par une partie pour connaître d'une procédure en matière de responsabilité parentale a été "acceptée expressément ou de toute autre manière non équivoque par toutes les parties à la procédure ", au sens de cette disposition, lorsque la partie défenderesse dans cette première procédure engage, ultérieurement, une seconde procédure devant la même juridiction et soulève, dans le cadre du premier acte qui lui incombe dans la première procédure, l'incompétence de cette juridiction » (par. 59).

Parce que dans cette solution l'accent tombe sur le comportement des parties dans la procédure, l'argumentation pourrait être utilisée aussi lors d'une discussion relative à l'efficacité d'une convention attributive de juridiction en matière de responsabilité parentale, antérieure au moment de la saisine de la juridiction. Lorsque les parties veulent la respecter, l'opérabilité de celle-ci est indubitable, sous la réserve du respect de l'intérêt supérieur de l'enfant (exigence qui devra être couramment analysée par l'instance). Les choses sont toutefois différentes lorsque l'une des parties conteste la compétence de la juridiction saisie sur cette base ; tenant compte tant de la formulation de l'article 12.3 - la compétence de la juridiction saisie doit avoir été acceptée à la date de la saisine de la juridiction -, que de l'argumentation de la Cour de justice dans l'arrêt $L$, probablement une opposition de la part de l'un des titulaires de la responsabilité parentale quant au déroulement du litige devant ladite juridiction serait de nature à priver d'effets la convention attributive de juridiction ${ }^{65}$.

1.3. Le deuxième arrêt relevant en la matière - Gogova - a été prononcé par la Cour en $2015^{66}$, suite à une question préjudicielle formulée par la Cour suprême de cassation de Bulgarie, intéressant l'interprétation du syntagme " acceptation expresse ou de toute autre manière non équivoque » dans un contexte particulier : absence de l'une des parties dans la procédure et sa représentation par un mandataire ad litem qui ne soulève pas un grief $d^{\prime}$ 'incompétence ${ }^{67}$. Dans son argumentation, la haute juridiction européenne a eu comme point de départ le fait que l'admission de l'autonomie de la volonté dans les litiges en matière de responsabilité parentale est une solution exceptionnelle, ainsi que les exigences de l'acceptation non équivoque de la compétence doivent être interprétées strictement (par. $41)^{68}$. L'acceptation devant provenir des parties au litige, une exigence minimale est la connaissance du commencement de celui-ci ; or, considère la Cour, bien que la connaissance de l'existence de la procédure ne vaille pas seule l'acceptation de la compétence, lorsque le 
défendeur a été absent de la procédure, qui n'a pas été notifiée, il ne peut pas être considéré comme avoir agréé la prorogation (par. 42). L'acceptation ne peut être déduite non plus du comportement du mandataire car, à défaut des contacts entre le défendeur et le mandataire, ce dernier ne peut pas extérioriser une volonté que soit celle du défendeur représenté. En décidant finalement que « l'article 12, paragraphe 3, sous b), du règlement $n^{\circ}$ 2201/2003 doit être interprété en ce sens que la compétence des juridictions saisies pour connaître d'une demande en matière de responsabilité parentale ne saurait être considérée comme ayant été " acceptée expressément ou de toute autre manière non équivoque par toutes les parties à la procédure", au sens de cette disposition, au seul motif que le mandataire ad litem représentant le défendeur, désigné d'office par ces juridictions au regard de l'impossibilité de notifier à ce dernier la requête introductive d'instance, n'a pas soulevé l'incompétence desdites juridictions $"$, la Cour offre une réponse non seulement prévisible ${ }^{69}$, mais aussi parfaitement compréhensible : lorsque la compétence se fonde sur la volonté des parties, le cadre légal existant doit garantir que celles-ci ont effectivement accepté cette compétence, ce qui n'est pas le cas lorsqu'une d'entre elles n'avait même pas connaissance de la procédure démarrée à son encontre.

1.4. Une autre discussion que peut soulever l'article 12 du règlement $2201 / 2003$ est celle de savoir si la prorogation envisagée peut être aussi tacite, selon le modèle consacré par l'article 26 du règlement Bruxelles I bis en matière civile et commerciale ${ }^{70}$.

À une première vue, les arguments dans le sens d'une réponse négative semblent majoritaires. Un premier obstacle à l'admission de la prorogation tacite serait représenté par les règles sur le rôle du juge : vue que selon l'article 17 du règlement, la juridiction saisie a la mission de contrôler sa compétence ${ }^{71}$, il résulte que la surveillance du respect des règles de compétence juridictionnelle édictées par le règlement n'est pas à la latitude des parties à travers l'exception de non-compétence, celle-ci ayant seulement un rôle subsidiaire à cet effet. Au contraire, les règles instituées ont un caractère impératif et la dérogation en peut être faite seulement dans les conditions prévues par les textes mêmes, ainsi qu'une simple comparution du défendeur ne devrait pas être suffisante pour la prorogation de la compétence. En même sens militerait tant le fait que la déduction de l'acceptation de la compétence du comportement des parties nécessiterait parfois, de la part des instances, une analyse ample des aspects de fait, de même que la position de la Cour de justice dans l'affaire L. c M., qui a exigé que l'acceptation de la compétence de la juridiction soit antérieure au commencement du litige. Enfin, cette solution restrictive figurait aussi dans la proposition originaire de règlement de 2002, qui précisait expressément que la comparution du défendeur ne vaut pas acceptation de la compétence.

Malgré ces arguments, la prise en considération d'un accord tacite pour la prorogation de la compétence dans les litiges en matière de responsabilité parentale ne nous semble pas une solution qui ne puisse pas être envisagée de lege $\operatorname{lata}^{72}$. La solution négative décrite plus haut est trop restrictive et contredit les analyses civilistes qui enseignent qu'une acceptation non équivoque peut revêtir aussi la forme d'une acceptation tacite. Par ailleurs, la doctrine a soutenu parfois une solution beaucoup plus libérale ${ }^{73}$, dont les avantages sont évidents en 
termes d'efficacité procédurale. Ni les arrêts de la Cour de justice ne sont pas univoques dans le sens du rejet d'une acceptation tacite de la compétence. En fait, dans l'arrêt $L$, la haute juridiction a précisé qu'il ne peut pas exister un accord exprès ou du moins univoque lorsque l'instance en cause est saisie seulement à l'initiative de l'une des parties à la procédure, tandis que l'autre, bien qu'introduisant une action séparée, conteste toutefois la compétence de la première instance ; per a contrario, lorsque la compétence n'est pas contestée et le défendeur comparaît, en formulant des conclusions sur le fond, la prorogation peut opérer. La présence du défendeur dans la procédure démontrerait sa volonté suffisante de voir l'affaire tranchée par les instances respectives ${ }^{74}$; la corrélation de sa volonté avec la volonté concordante du demandeur, déduite de l'introduction de la requête devant ces instances précises, conduirait à l'existence d'une acceptation "de toute autre manière non équivoque " de la prorogation. Par ailleurs, une lecture atténuée des exigences de l'article 17, conduisant au même résultat, pourrait-elle aussi être envisagée : l'instance doit vérifier sa compétence et une démarche minimale serait celle de mettre la question au débat des parties; si le défendeur ne soulève pas des objections et les autres conditions de l'article 12 sont remplies, la prorogation pourra être acceptée.

À défaut d'une prise de position de la Cour de justice, la solution peut être discutée. Éventuellement, pour un peu plus de clarté en la matière, dans le contexte des réformes futures, il pourrait être introduite dans le règlement une règle de nature à résoudre ce problème. À notre avis, l'admission de la prorogation tacite de la compétence est une alternative parfaitement acceptable, la garantie du consentement réel du défendeur pouvant être réalisée concrètement à travers un correctif similaire à celui prévu dans le règlement 1215/2012 pour la prorogation tacite de la compétence dans les litiges impliquant des parties vulnérables $^{75}$ : avant de se déclarer compétentes, les juridictions saisies devraient s'assurer que le défendeur (la partie vulnérable) est informé sur son droit de contester la compétence et sur les conséquences de sa comparution.

\section{Le respect de l'intérêt supérieur de l'enfant}

2.1. La deuxième condition essentielle pour l'admission de la prorogation volontaire de la compétence concerne le respect de l'intérêt supérieur de l'enfant : tant l'article 12.1, que l'article 12.3 du règlement la mentionnent expressément, fait de nature à encourager les juridictions à réaliser sa vérification systématique et de motiver leurs décisions de ce point de vue $^{76}$.

Parce que l'intérêt supérieur de l'enfant reste une condition indispensable pour la prorogation, l'autonomie de la volonté pourra supplémentairement être censurée. Même si dans l'arrêt $E$, la Cour de justice affirmait que « la prorogation d'une compétence acceptée par les titulaires de la responsabilité parentale d'un jeune enfant pour une procédure spécifique peut être considérée comme étant dans l'intérêt supérieur de cet enfant » (par. 46), consacrant une présomption indirecte de respect de cet intérêt une fois l'accord réalisé, en fait, entre les 
deux conditions - l'accord et le respect de l'intérêt supérieur - ne peut pas être mis un signe d'égalité. Généralement, le consensus des parties se traduit dans une solution favorable plus largement aux intérêts de l'enfant ${ }^{77}$. Toutefois, tel peut n'être pas toujours le cas en pratique, et par la mention séparée des deux exigences, le législateur en tient compte adéquatement : ainsi, sur la base du texte légal, même si les deux parents sont d'accord avec la prorogation de la compétence, des juridictions pourraient censurer leur accord, lorsque celui-ci ne soutient pas également l'intérêt de l'enfant ; les parents, les titulaires de la responsabilité parentale et éventuellement les autres parties dans le litige n'ont pas la latitude de décider souverainement quel est cet intérêt, leurs décisions, y compris du point de vue procédural, restent sous le contrôle des juges ${ }^{78}$.

2.2. $\mathrm{Si}$, selon la Cour de justice, l'exigence doit être vérifiée au cas par $\operatorname{cas}^{79}$, la réglementation légale du contenu concret de cet intérêt supérieur est vague, ce qui fait que son interprétation suscite des difficultés et entraîne des solutions non-uniformes en pratique. Dans l'article 12.4 du règlement, le législateur européen apporte une seule précision concernant le respect de l'intérêt supérieur de l'enfant en cas de prorogation de compétence, limitée à une hypothèse particulière : celle de la résidence habituelle de l'enfant sur le territoire d'un État non européen qui n'est pas partie contractante à la Convention de La Haye. En cette hypothèse, la prorogation opérée sur la base de l'article 12 « est présumée être dans l'intérêt de l'enfant, notamment lorsqu'une procédure s'avère impossible dans l'État tiers concerné » ; dans la Convention de La Haye de 1996, il n'y a pas une telle règle. En raison de sa spécificité et de son caractère très circonstanciel, la solution ne peut pas être généralisée pour les hypothèses de sélection entre plusieurs instances des différents États membres (où le déroulement des procédures est possible).

La doctrine s'est arrêtée sur cette notion - l'intérêt supérieur de l'enfant -, sans que les solutions offertes concernent strictement le problème de la procédure ${ }^{80}$. Les indications de la Cour de justice sur les aspects pouvant être pris en compte dans l'évaluation ne sont pas toujours révélatrices. Par exemple, dans les arrêts $A$ et Mercredi, la Cour a précisé que pour l'interprétation du concept "résidence habituelle " doit être tenu compte de l'intérêt supérieur de l'enfant, ce qui implique la vérification de la proximité étroite entre l'instance et l'endroit où le mineur est intégré du point de vue familial et social. Similairement, dans l'arrêt $L c$. $M$, elle a lié l'intérêt supérieur de l'enfant du lien étroit entre celui-ci et la juridiction saisie $^{81}$. Toutefois, en pratique les deux aspects ne devraient pas être confondus. Au-delà du fait que le législateur les a prévus séparément ${ }^{82}$, on croit qu'un accent trop grand sur la (meilleure) proximité entre la juridiction compétente et le lieu où se déroule la vie du mineur, comme élément essentiel d'identification de son intérêt supérieur, dépasse l'esprit du texte ; étant donné que le critère de la résidence habituelle, prévu à l'article 8 du règlement, est considéré exprimer de façon principielle les liens les plus étroits, la prorogation arriverait à être admise seulement rarement, l'article 12 étant ainsi privé de son effet utile ${ }^{83}$.

2.3. L'intérêt supérieur de l'enfant a été évoqué par la Cour de justice aussi dans un arrêt d'octobre 2016, Child and Family Agency ${ }^{84}$, dans le contexte d'une question préliminaire relative à l'article 15 du règlement. La Cour a précisé que l'instance saisie (sur la base de 
l'article 8) doit s'assurer, en tenant compte des circonstances concrètes de l'affaire, que le transfert de la compétence vers une autre instance ne risque pas d'avoir une incidence préjudiciable sur la situation de l'enfant visé et, à cet effet, elle "doit évaluer l'éventuelle incidence négative qu'un tel renvoi pourrait avoir sur les rapports affectifs, familiaux et sociaux de l'enfant concerné par l'affaire ou sur la situation matérielle de celui-ci » (par. 58-59). Transposables dans le contexte d'une prorogation volontaire de la compétence, les indices offerts restent toutefois vagues et, corrélativement, assez déficitaires dans la perspective de l'assurance de la prévisibilité et de la certitude juridique pour les personnes intéressées. Plus détaillées et utiles apparaissent les précisions de l'avocat général Wathelet, dans ses conclusions $^{85}$ : il suggère qu'en vue d'apprécier la capacité d'une instance d'apprécier une affaire dans l'intérêt supérieur de l'enfant peuvent être pris en compte des éléments tels « la langue de procédure, la disponibilité des éléments de preuve pertinents relatifs, par exemple, à la capacité d'éducation et d'entretien du ou des parents, la possibilité de citer les témoins utiles et la probabilité que ceux-ci comparaissent, la disponibilité de rapports médicaux et sociaux et la possibilité de les actualiser le cas échéant, voire le délai dans lequel la décision sera rendue " (par. 95) ${ }^{86}$; parce que l'intérêt supérieur de l'enfant est le mieux préservé lorsque la compétence revient aux juridictions de l'État de la résidence habituelle de l'enfant et aussi parce que certains documents utiles à l'appréciation de l'affaire pourraient être obtenus plus facilement par la coopération entre les juridictions, l'avocat général suggère en même temps la prise en considération de "l'incidence que pourrait avoir sur le bien-être physique et moral [de l'enfant] le déplacement inhérent à un renvoi de l'affaire à une juridiction située dans un autre État membre » que celui dans lequel l'enfant a sa résidence habituelle (par. 96). Bien que fortement nuancées par le contexte spécifique de la question préliminaire (qui visait les conditions de la mise en œuvre du mécanisme exceptionnel de forum non conveniens de l'article 15), ses indications peuvent être généralisées et pris en compte aussi pour l'interprétation de l'exigence corrélative de l'article 12 du règlement.

Conclusions. Les solutions retenues par le législateur européen dans le règlement 2201/2003 reflètent largement des considérants contradictoires quant à l'admissibilité de l'autonomie de la volonté dans les litiges internationaux de divorce, séparation de corps ou annulation du mariage et responsabilité parentale. D'un côté, pour le contentieux matrimonial, la volonté de préserver des conceptions étatiques particulières a imposé des limitations plus que sévères en ce qui concerne la liberté des époux de choisir la juridiction compétente. La solution n'est pas toutefois cohérente ni avec celle promue dans le plan des conflits de lois, ni avec la politique qui inspire l'article 3 du règlement (la multiplication des critères de compétence juridictionnelle, parmi lesquels le demandeur peut choisir celui qui lui est plus convenable) ; dans les conditions d'un libéralisme accentué aussi dans le plan du droit substantiel interne, l'admission dans la future réforme d'une autonomie encadrée serait de nature à mieux servir les intérêts européens - la correction des uns des inconvénients de l'actuelle règle de l'article 3 , en soutenant en même temps la libre circulation, l'accès à la justice, la prévisibilité pour les personnes 
impliquées, mais aussi l'efficience économique. D’un autre côté, pour le contentieux de la responsabilité parentale, le législateur européen a été plus ouvert aux solutions volontaristes : sous la condition de l'existence d'un lien suffisant entre l'État de la juridiction saisie et l'enfant et du respect de l'intérêt supérieur de ce dernier, l'autonomie de la volonté est admissible. Cela promeut le consensualisme aussi pour la solution des problèmes de fond, reflète une plus grande ouverture pour les besoins des parties intéressées et apporte un plus de flexibilité dans le jeu des règles instituées, sans que la proximité soit sacrifiée. Certes, les solutions sont perfectibles et dans le contexte des réformes futures, il pourrait être envisagé, y compris sur la base des arrêts récents de la Cour de justice, la clarification des conditions d'extériorisation de la volonté des parties (spécialement du point de vue de l'accord tacite), afin de renforcer la sécurité juridique, la certitude et la prévisibilité pour tous ceux intéressés. Dans les deux domaines, comme toujours, le dernier mot reste toutefois au législateur et il est attendu avec intérêt.

\footnotetext{
* Chargée de cours, Faculté de Droit, Univ. Babes-Bolyai, Cluj-Napoca, Romania. La présente étude a été réalisée avec le support financier de I'Université Babeş-Bolyai, à travers le Grant de recherche GTC - 31804/2016.

1 Règlement (CE) $n^{\circ} 2201 / 2003$ du Conseil du 27 novembre 2003 relatif à la compétence, la reconnaissance et l'exécution des décisions en matière matrimoniale et en matière de responsabilité parentale, JO L 338, 23.12.2003; Règlement (UE) n 1259/2010 du Conseil du 20 décembre 2010 mettant en œuvre une coopération renforcée dans le domaine de la loi applicable au divorce et à la séparation de corps, JO L 343, 29.12.2010; Règlement (CE) $n^{\circ}$ 4/2009 du Conseil du 18 décembre 2008 relatif à la compétence, la loi applicable, la reconnaissance et l'exécution des décisions et la coopération en matière d'obligations alimentaires, JO L 7, 10.1.2009; Règlement (UE) n²016/1103 du Conseil du 24 juin 2016 mettant en œuvre une coopération renforcée dans le domaine de la compétence, de la loi applicable, de la reconnaissance et de l'exécution des décisions en matière de régimes matrimoniaux, JO L 183, 8.7.2016; Règlement (UE) n² 2016/1104 du Conseil du 24 juin 2016 mettant en œuvre une coopération renforcée dans le domaine de la compétence, de la loi applicable, de la reconnaissance et de l'exécution des décisions en matière d'effets patrimoniaux des partenariats enregistrés, JO L 183, 8.7.2016.

${ }^{2}$ Règlement (CE) n 1347/2000 relatif à la compétence, la reconnaissance et l'exécution des décisions en matière matrimoniale et en matière de responsabilité parentale des enfants communs, JOCE L $160,30.06 .2000$.

${ }^{3}$ Le fondement juridique de la technique de consolidation consolidée est représenté par les articles 20 TUE et 326-334 TFUE. Le recours à cette modalité de légiférer a comme conséquence le fait que le règlement 1259/2010 n'uniformise pas les règles de conflit en matière de divorce ou séparation de corps de tous les États européens, mais seulement celles des États ayant participé à son adoption ou qui ont adhéré ultérieurement à la coopération consolidée instituée. La situation mise à jour des
} 
États liés est disponible sur le site www.e-justice.europa.eu. Sur la complexité entrainée, v. P. Hammje, " Le nouveau règlement (UE) n 1259/2010 du Conseil du 20 décembre 2010 mettant en œuvre une coopération renforcée dans le domaine de la loi applicable au divorce et à la séparation de corps ", Rev. crit. DIP, 2011, p. 291, spéc. p. 295.

${ }^{4}$ Pour la Roumanie, il s'agit de l'article 2588 C. civ., qui renvoie aux lois qui gouvernent les exigences légales pour la conclusion du mariage, dont la désignation sera faite selon les articles 2586 et 2587 C. civ.

${ }^{5}$ Pour la Roumanie, l'article 2611 C. civ. renvoie à la Convention de La Haye du 19 octobre 1996 relative à la protection des mineurs, qui distingue entre la loi applicable à l'attribution et à l'exercice de l'autorité parentale, d'une part, et la loi applicable à la prise des mesures de protection, d'un autre.

${ }^{6}$ Pour une analyse récente de la portée de l'intrusion de la volonté des parties en droit international privé de la famille (conflits de lois), v. B. Bourdelois, "Relations familiales internationales et professio juris ", in Les relations privées internationales: mélanges en l'honneur du Professeur Bernard Audit, LGDJ, 2014, p. 137 ; C.I. Nagy, „What Functions May Party Autonomy Have in International Family and Succession Law ? An EU Perspective", Nederlands Internationaal Privaatrecht (NIPR), 2012, Vol. 30, No. 4, p. 576-586. V. déjà J.Y. Carlier, Autonomie de la volonté et statut personnel. Etude prospective de droit international privé, Bruylant, Bruxelles, 1992 ; P. Gannagé, " La pénétration de l'autonomie de la volonté dans le droit international privé de la famille ", Rev. crit. DIP, 1992, p. 425-454 ; E. Jayme, "Identité culturelle et intégration : le droit international privé postmoderne ", RCADI, 1995, t. 251, spéc. p. 54-55 et 153.

${ }^{7}$ Cette tendance d'extension du rôle de l'autonomie de la volonté est soutenue par un phénomène similaire dans le plan du droit substantiel interne; dans le sens que l'autodétermination des individus est un élément définitoire des réglementations actuelles en droit de la famille, $v$. par exemple $P$. Franzina, "The law applicable to divorce and legal separation ", Cuadernos de Derecho Transnacional, 2011 , vol. 3, no 2, p. 85, spéc. p. 108, nº. 44. Le droit européen reflète lui-aussi la même tendance : au niveau des États membres du Conseil de l'Europe, la jurisprudence de la Cour européenne des droits de l'homme relative à l'article 8 soutient comme composante du droit à la vie privée et familiale le droit d'une personne de prendre des décisions en ce qui concerne, par exemple, sa vie sexuelle (CEDH, 19 février 1997, Laskey c. Royaume Uni, par. 44), la procréation (CEDH, 10 avril 2007, Evans c. Royaume Uni, par.7) ou son identité personnelle (CEDH, 11 juillet 2002, Goodwin v c. Royaume Uni, par. 90).

${ }^{8}$ Sur cette progression de l'autonomie de la volonté au-delà de la sphère des obligations, à des aspects personnels, v. par exemple E. Jayme, " Party autonomy in international family and succession law : new tendencies ", Swiss Yearbook of PIL, 2009, vol 11, p. 1 et s. ; B. Ancel, H. Muir Watt, " Aliments sans frontières. Le règlement $\mathrm{CE} \mathrm{n}^{\circ} 4 / 2009$ du 18 décembre 2008 relatif à la compétence, la loi applicable, la reconnaissance et l'exécution des décisions et la coopération en matière d'obligations alimentaires ", Rev. crit. DIP, 2010, p. 457, spéc. p. 467, n 9. Pour les solutions concrètes, v. les articles 4 et 5 du règlement $4 / 2009$, qui admettent l'élection expresse ou tacite de la juridiction compétente pour les litiges nés ou éventuels en matière d'obligations d'entretien ; une limitation importante, justifiée par l'objectif d'assurer une protection adéquate à l'intérêt supérieur de l'enfant, a été toutefois prévue pour les litiges relatifs aux obligations alimentaires dont le créancier est un enfant âgé de moins de 18 ans (la compétence devant être établie sur la base des règles objectives). V. aussi les solutions du règlement 650/2012 du Parlement européen et du Conseil du 4 juillet 2012 relatif à la compétence, la loi applicable, la reconnaissance et l'exécution des décisions, et l'acceptation et 
l'exécution des actes authentiques en matière de successions et à la création d'un certificat successoral européen, OJ L 201, 27.7.2012 (en particulier, les articles 5 et 9 sur la prorogation volontaire de compétence). V. aussi les règlements 2016/1103 et 2016/1104, qui encadrent la liberté des parties de choisir la juridiction compétente de sorte que les objectifs de bonne administration de la justice, proximité, coïncidence entre la compétence juridictionnelle et la compétence législative puissent être au mieux réalisés ( $v$. spécialement l'article 7 , de même que les articles 8 et 5.2 de chacun de ces textes).

${ }^{9}$ Les solutions volontaristes ne sont toutefois une panacée, car des fois l'accord peut manquer, vu la nature délicate des problèmes soulevés par le domaine familial.

${ }^{10}$ Par ailleurs, même dans le plan des conflits de lois il est accepté que, lorsque l'option des parties est limitée à un éventail de lois avec lesquelles la situation présente des liens, l'autonomie de la volonté n'agit plus comme un promoteur de la concurrence législative qui ébranle les politiques impératives étatiques, mais apporte, au contraire, un plus de stabilité et de prévisibilité pour les personnes intéressées, dont la situation est déjà fragilisée par le jeu des conflits de lois; l'autonomie de la volonté devient ainsi un instrument de coordination des systèmes juridiques - v. $\mathrm{H}$. Gaudemet-Tallon, Le pluralisme en DIP : richesses et faiblesses (le funambule et l'arc-en-ciel): cours général, RCADI, 2005, p. 292.

${ }^{11}$ Toutefois, le droit de la famille connait aussi des nuances importantes de "privatisation " (étant admis le divorce par consentement mutuel, étant offertes des options plus larges quant au régime matrimonial ou étant permises des ententes sur les obligations alimentaires, par exemple), qui pourraient se répercuter sans trop graves inconvénients sur les problèmes procéduraux.

12 Il est applicable dans tous les États membres de l'Union européenne (à l'exception du Danemark), à partir du $1^{\mathrm{er}}$ mars 2005. Pour la Roumanie et Bulgarie, États qui ont adhéré à l'Union en 2007, le

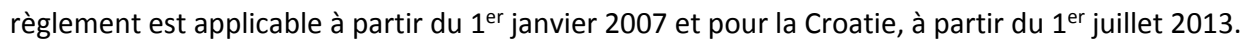

${ }^{13}$ En détail sur les objectifs poursuivis par le législateur européen en la matière, v. par exemple B. Ancel, H. Muir-Watt, "La désunion européenne : le règlement dit Bruxelles II ", RCDIP, 2001, p. 403 , spéc. p. 411 et s., $n^{\circ} 5$.

${ }^{14}$ L'articulation entre les normes européennes et celles nationales est réglée par les articles 6 et 7 du règlement, avec les précisions apportées par la Cour de justice dans l'arrêt Sundelind Lopez (CJCE, 29 novembre 2007, C-68/07) ; vu la priorité qui doit être accordée aux normes européennes, l'intervention des normes nationales de procédure civile internationale est fortement limitée. Le règlement Bruxelles 2 bis se substitue aux normes nationales de compétence, auxquelles est réservée seulement une place résiduelle.

${ }^{15}$ Sur celles-ci, v. R. Hausmann, "Article 3 ", in S. Corneloup (coord.), Droit européen du divorce European Divorce Law, Lexis Nexis, 2013, p. 238 et s. ; A. Borras, "Article 3 ", in U. Magnus, P. Mankowski (eds.), Brussels II bis Regulation, Sellier, 2012, p. 87 et s. ; v. également B. Ancel, H. Muir Watt, "L'intérêt supérieur de l'enfant dans le concert des juridictions : le règlement Bruxelles II bis ", Rev. crit. DIP, 2005, p. 569, spéc. p. 577-579, n 8.

${ }^{16}$ V. B. Ancel, H. Muir Watt, "L'intérêt supérieur... », op. cit., nº 8-9, p. 577-579.

${ }^{17}$ Malheureusement, les interprétations des juridictions nationales n'en tiennent pas toujours compte et elles sont de plus en plus diverses; cela affecte l'application uniforme du règlement et accroit l'insécurité juridique. 
${ }^{18}$ CJCE, 2 avril 2009, C-523/07, A; CJUE, 22 décembre 2010, C-497/10 PPU, Mercredi; v. aussi CJUE, 6 octobre 2015, aff. C-404/14, Marie Matouskova, D. 2016, p. 1045, obs. H. Gaudemet-Tallon et F. Jault-Seseke.

${ }^{19}$ Les règles de procédure civile internationale stipulées par le règlement ne sont obligatoires que pour les instances des États membres de l'Union dans lesquels le règlement est applicable; la compétence internationale des instances des États tiers, dans les litiges impliquant des citoyens européens, sera établie en chaque cas particulier selon les règles de procédure civile de l'État de la juridiction saisie.

${ }^{20}$ Sur l'interprétation de la notion « demande commune », v. R. Hausmann, op. cit., p. 246-247, no $18-$ 19.

${ }^{21}$ V. R. Hausmann, op. cit., p. $238, \mathrm{n}^{\circ} 2$.

22 Proposition de Règlement du Conseil modifiant le règlement (CE) $n^{\circ} 2201 / 2003$ en ce qui concerne la compétence et instituant des règles relatives à la loi applicable en matière matrimoniale, COM/2006/0399 (17.7.2006). Sur celle-ci, v. I. Barrière-Brousse, "La révision du règlement Bruxelles II bis : perspectives communautaires sur les désunions internationales ", D. 2008, p. 625.

${ }^{23}$ Celui-ci était formulé comme suit : "Choix de la juridiction par les parties dans les procédures de divorce et de séparation de corps 1 . Les conjoints peuvent convenir qu'une juridiction ou les juridictions d'un État membre auront compétence pour statuer dans une procédure de divorce ou de séparation de corps qui les oppose, à condition qu'ils aient des liens étroits avec ledit État membre en raison du fait que: I'un des critères de compétence énumérés à l'article 3 s'applique, ou il s'agit du lieu de la dernière résidence habituelle commune des conjoints pendant une durée d'au moins trois ans, ou l'un des conjoints est ressortissant de cet État membre ou, dans le cas du Royaume-Uni et de l'Irlande, a son «domicile» sur le territoire d'un de ces deux États membres. 2. La convention attributive de compétence doit être formulée par écrit et signée par les deux conjoints au plus tard au moment de la saisine de la juridiction ».

24 L'autonomie de la volonté n'était pas admise pour les litiges d'annulation du mariage. Cette limitation semble compréhensible, car les problèmes de fond en discussion reflètent des conceptions particulières des États sur le mariage et sa validité ; or, faire dépendre l'efficacité de celles-ci de la libre volonté des personnes privées apparaissait totalement inopportun.

${ }^{25}$ La solution était d'autant plus remarquée que, en considération du caractère indisponible des droits en discussion, les nomes étatiques de compétence juridictionnelle avaient un caractère impératif v. I. Barrière-Brousse, op. cit., p. $629, \mathrm{n}^{\circ} 19$.

${ }^{26}$ Cette solution est contraire à la celle retenue généralement en matière civile et commerciale par (l'actuel) article 26 du règlement 1215/2012 concernant la compétence judiciaire, la reconnaissance et l'exécution des décisions en matière civile et commerciale (refonte) (Bruxelles I bis), JO L 351, 20.12.2012.

${ }^{27}$ Ces efforts ont été finalisés avec l'adoption du règlement 1259/2010, qui trahit pourtant certains des objectifs initiaux du législateur européen : ce règlement ne se superpose pas parfaitement au règlement 2201/2003 ni du point de vue géographique, étant obligatoire seulement pour une partie des États membres, ni du point de vue matériel, en excluant la problématique de l'annulation du mariage de son champ d'application.

${ }^{28} \mathrm{COM}(2014) 225$ final. 
${ }^{29}$ Deloitte, Coffey, Study on the assessment of Regulation (EC) No 2201/2003 and the policy options for its amendment (May 2015), disponible à l'adresse http://ec.europa.eu/justice/civil/files/bxl_iia_final_report_evaluation.pdf.

${ }^{30}$ Deloitte, Coffey, op. cit., p. 25, p. 29.

${ }^{31}$ Deloitte, Coffey, op. cit., annexe 1, p. 21, disponible à l'adresse : http://ec.europa.eu/justice/civil/files/bxl_iia_final_report_analtical_annexes.pdf.

${ }^{32}$ Commission européenne, „Proposition de Règlement du Conseil relatif à la compétence, la reconnaissance et l'exécution des décisions en matière matrimoniale et en matière de responsabilité parentale, ainsi qu'à l'enlèvement international d'enfants (refonte), COM(2016) 411 final.

${ }^{33} \mathrm{~V}$. ainsi l'article 5 du règlement 1259/2010 (Roma III). Deux observations sont nécessaires : d'un côté, en raison de la technique suivie pour son adoption (la coopération consolidée), ce règlement n'est pas en vigueur dans tous les États membres; d'un autre côté, la solution de l'admission de l'autonomie de la volonté retenue dans l'article 5 du règlement était toutefois novatrice au niveau européen, seulement l'Allemagne, la Belgique et les Pays-Bas consacrant antérieurement des règles de conflit subjectives en la matière - v. P. Franzina, op. cit., spéc. p. 108, n ${ }^{\circ} 42$.

${ }^{34}$ En dépit du champ d'application territorial et matériel large du règlement, les règles actuelles n'arrivent pas à garantir l'accès à un juge d'un Etat membre justice pour tous les citoyens européens. Ainsi, pour un couple de citoyens européens de nationalités différentes vivant dans un (des) pays tiers, pour un citoyen européen marié avec un ressortissant d'un état tiers et vivant dans un (des) Etat(s) tiers ou pour les époux homosexuels de nationalités différentes et vivant dans un Etat (membre) ou leur mariage n'est pas reconnu, l'accès à la justice peut s'avérer délicat. L'admission de l'autonomie de la volonté serait de nature à corriger ce problème (sans qu'elle soit toutefois le seul mécanisme disponible à cet effet).

35 V. Deloitte, Coffey, op. cit., p. 38, soutenant que l'admission des conventions attributives de juridiction en la matière serait de nature à protéger la partie la plus faible du point de vue économique devant un forum shopping démarré par le partenaire qui a eu des ressources suffisantes pour obtenir des conseils juridiques adéquats sur les juridictions devant lesquelles il pourrait obtenir un traitement plus favorable à ses intérêts.

${ }^{36}$ Le règlement 1347/2000 était applicable aux litiges en matière de responsabilité parentale seulement lorsque ceux-ci concernaient les enfants communs au couple (et non également pour les litiges concernant les enfants naturels ou les enfants d'un seul époux) et étaient connexes à une procédure en cours relative au lien matrimonial (v. le considérant 11 et l'article 1.1.b) du règlement 1347/2000); de ce point de vue, il a été considéré insatisfaisant et à l'initiative de la France, son domaine d'application a été élargi.

${ }^{37}$ Bien que l'article article 12 est une exception à l'article 8 (compétence des instances de l'État de la résidence habituelle de l'enfant), les deux critères de compétence peuvent fonctionner alternativement - v. E. Pataut, "Article 12 », in U. Magnus, P. Mankowski, op. cit., p. 148, $\mathrm{n}^{\circ}$ 9. Sur la qualification et sur la mise en œuvre du critère de la résidence habituelle, v. E. Gallant, "Réflexions sur la résidence habituelle des enfants des couples désunis ", in Mélanges en l'honneur du Professeur Pierre Mayer : liber amicorum, LGDJ, 2015, p. 241-253.

${ }^{38}$ Même si en droit comparé la solution est très fréquente pour les litiges internes, la Convention de La Haye de 1961 sur la protection des mineurs met en évidence une tendance opposée, celle de séparation entre les litiges matrimoniaux et ceux relatifs à la responsabilité parentale $-v$. E. Pataut, op. cit, p. 147, $\mathrm{n}^{\circ} 4$. 
${ }^{39}$ V. par exemple en ce sens CJUE, $1^{\text {er }}$ octobre 2014, E C. B, C-436/13, par. $41-42$ (dans lequel est invoqué le considérant 12 du préambule) et CJUE, 12 novembre 2014, C-656/13, L C. $M$, par. 48; V. aussi CJUE, 2 avril 2009, C-523/07, A, par. 35); CJUE, 23 décembre 2009, C-403/09 PPU, Detiček, par. 35; CJUE, 22 décembre 2010, C-497/10 PPU, Mercredi, par. 46; CJUE, 15 juillet 2010, C-256/09, Purrucker, par. 91.

${ }^{40}$ V. B. Ancel, H. Muir-Watt, "L'intérêt supérieur... ", op. cit., p. 580, nº 11.

${ }^{41}$ Cette alternative se retrouvait aussi à l'article 10 de la Convention de La Haye de 1996, qui a servi comme source d'inspiration pour le législateur européen. Sa reprise atteste la volonté du législateur d'assurer une articulation cohérente des deux textes internationaux. En tenant compte des dispositions de l'article 61 du règlement, la compétence des juridictions des États membres européens sera établie selon le règlement (y compris son article 12) lorsque l'enfant aura sa résidence habituelle dans un État membre ou dans un État tiers qui n'est pas partie à la Convention de La Haye. Lorsque l'enfant aura sa résidence habituelle dans un État non-européen, partie à la Convention de La Haye, les dispositions de l'article 10 de ce dernier texte vont être suivies (v. infra).

${ }^{42}$ Sur l'extension opérée par le règlement Bruxelles 2 bis de ce point de vue, $v$. W. Pintens, "Article 1 », in U. Magnus, P. Mankowski (eds.), op. cit., p. 73-74, $\mathrm{n}^{\circ} 60$.

${ }^{43}$ Toutefois, même avec cette limitation (mais dans le contexte de la multiplication des critères de compétence juridictionnelle en matière de contentieux matrimonial), la solution ne garantit pas un lien très étroit entre l'enfant et l'État de l'instance saisie - v. B. Ancel, H. Muir-Watt, "L'intérêt supérieur... ", op. cit., p. 586, $\mathrm{n}^{\circ} 16$.

${ }^{44}$ E. Pataut, op. cit., p. 150, n' 20-21. À notre avis, rien n'empêche en ces cas les parents et les titulaires de la responsabilité parentale à se prévaloir de l'article 12.3 du règlement, à condition toutefois de prouver le lien étroit exigé par l'article 12.3. a) entre la situation de l'enfant et l'État de la juridiction élue.

${ }^{45}$ V. aussi CJUE, 15 février 2017, C-499/15, W et V c. X : « ainsi que M. l'avocat général l'a relevé aux points 43 à 49 de ses conclusions, [...] les juridictions ayant statué en matière de divorce, en l'occurrence les juridictions lituaniennes, ne bénéficient pas dans une affaire telle que celle au principal d'une prorogation de compétence. Quand bien même la compétence de ces juridictions aurait été acceptée expressément ou de toute autre manière non équivoque par $X$, conformément à l'article 12 , paragraphe 1 , sous b), du règlement $n^{\circ} 2201 / 2003$, cette compétence $a$, en tout état de cause, pris fin dès que la décision faisant droit à la demande en divorce et statuant sur la responsabilité parentale est passée en force de chose jugée, conformément à l'article 12 , paragraphe 2 , sous a) et b) de ce règlement » (par. 68).

${ }^{46}$ L'article 10 de la Convention de La Haye : " 1 . Sans préjudice des articles 5 à 9 , les autorités d'un État contractant, dans l'exercice de leur compétence pour connaître d'une demande en divorce ou séparation de corps des parents d'un enfant résidant habituellement dans un autre État contractant, ou en annulation de leur mariage, peuvent prendre, si la loi de leur État le permet, des mesures de protection de la personne ou des biens de l'enfant, a) si, au commencement de la procédure, I'un des parents réside habituellement dans cet État et que l'un d'eux ait la responsabilité parentale à l'égard de l'enfant, et b) si la compétence de ces autorités pour prendre de telles mesures a été acceptée par les parents, ainsi que par toute autre personne ayant la responsabilité parentale à l'égard de l'enfant et si cette compétence est conforme à l'intérêt supérieur de l'enfant. 2. La compétence prévue au paragraphe premier pour prendre des mesures 
de protection de l'enfant cesse dès lors que la décision faisant droit ou rejetant la demande en divorce, séparation de corps ou annulation du mariage est devenue définitive ou que la procédure a pris fin pour un autre motif $"$.

${ }^{47}$ V. E. Pataut, op. cit., p. $148, n^{\circ} 13$.

${ }^{48}$ La solution, qui n'a pas un correspondant dans l'article 10 de la Convention de La Haye de 1996, se remarque par sa nouveauté.

${ }^{49}$ Dans le sens que le législateur n'a pas voulu instituer des présomptions, mais simplement offrir des exemples, v. Th. Rauscher, "Parental Responsibility Cases under the new Council Regulation Brussels IIA ॥, The European Legal Forum, 1-2005, p. 37, spéc. p. 41.

50 Toutefois, dans l'arrêt de 12 novembre 2014, C-656/13, L c. M, qui sera présenté infra, la Cour de justice a lié l'intérêt supérieur de l'enfant du lien étroit entre l'enfant et l'instance saisie.

${ }^{51}$ Sur ces discussions, v. B. Ancel, H. Muir-Watt, "L'intérêt supérieur... ", op. cit., p. 587-589, n 17.

52 Commission européenne, Guide pratique pour l'application du règlement Bruxelles II bis (spéc. $\mathrm{p}$. 32), disponible à l'adresse http://ec.europa.eu/justice/civil/files/brussels_ii_practice_guide_fr.pdf.

53 CJUE, 12 novembre 2014, C-656/13, L c. M, Rev. Crit. DIP, 2015, p. 667, note E. Gallant ; V. Egéa, "Les prorogations de compétence dans le règlement Bruxelles II bis : d'utiles précisions ", Rev. Trim. Dt. Eur., 2015, p. 383. Le litige portait sur la situation des deux enfants, ressortissants tchèques, qui sont nés et qui ont vécu de façon permanente en République Tchèque, avec leurs parents, un couple non marié. Du 2010 jusqu'au 2012, suite à la séparation des parents, les enfants ont habité alternativement avec leur mère $(L)$, en Autriche, et avec leur père $(M)$, en république Tchèque. En mai 2012, la mère a enregistré le domicile des enfants en Autriche. En octobre 2012, $M$ a saisi les instances tchèques avec une demande concernant l'organisation des relations entre les parents et les enfants, suivie, quelques jours plus tard, par une demande introduite devant les mêmes instances par $L$, concernant la custodie des enfants et une pension alimentaire. Ultérieurement, après avoir réitéré sa demande devant les juridictions autrichiennes, $L$ a contesté la compétence de la juridiction tchèque. Le litige est arrivé jusqu'à la Cour suprême qui a décidé de saisir la Cour de justice de l'UE avec deux questions préliminaires relatives à l'article 12.3 du règlement; la première concerne précisément le problème de savoir si la prorogation de compétence visée par ce texte peur opérer aussi lorsque entre les parents (titulaires de la responsabilité parentale) n'existe pas un litige de divorce ou toute autre procédure pendante ayant un autre objet; les questions préliminaires sont reproduites au par. 31 de l'arrêt.

${ }^{54}$ V. B. Ancel, H. Muir-Watt, "L'intérêt supérieur... ", op. cit., p. 589, $\mathrm{n}^{\circ} 18$. Dans le sens que la prorogation peut opérer seulement lorsque les instances sont déjà saisies avec une autre procédure, sans que celle-ci soit nécessairement matrimoniale, v. R. Baratta, "II regolamento comunitario sul diritto internazionale privato della famiglia ", in P. Picone (coord.), Diritto internazionale privato e diritto comunitario, Padova, 2004, p. 177. Le problème qui fait l'objet de la question préliminaire a été discuté aussi devant les juridictions roumaines, qui ont opté pour une interprétation restrictive du texte : par exemple, dans une affaire de 2012, la Cour d'Appel Bucarest s'est prononcée avec clarté dans le sens de l'existence d'une action connexe pendante devant I'instance convenue par les parties pour statuer en matière de responsabilité parentale; considérant que l'interprétation de l'article 12.3 doit se faire par corroboration avec l'article 12.1 du règlement, elle a décidé que, lorsque entre les parties n'existe pas une autre procédure en cours, la prorogation visée par l'article 12.3 ne peut pas opérer (C. Ap. Bucarest, $3^{\text {ème }}$ ch. civ., déc. $n^{\circ} 1054$, séance publique du 7.06.2012). 
${ }^{55} \mathrm{~V}$. les références citées par E. Gallant, Note précitée, p. 678-679, $\mathrm{n}^{\circ} 8$.

${ }^{56}$ La solution a été indirectement confirmée dans l'arrêt Gogova (CJUE, 21 octobre 2015, C-215/15 ; v. infra).

57 Commission européenne, "Proposition de règlement du Conseil relatif à la compétence, la reconnaissance et l'exécution des décisions en matière matrimoniale et en matière de responsabilité parentale, ainsi qu'à l'enlèvement international d'enfants (refonte) ", COM (2016) 411 final.

${ }^{58}$ CJUE, 1 octobre 2014, E c. B, C-436/13. Le litige entre le père espagnol (E) et la mère anglaise (B) concernait l'établissement de la résidence de leur enfant et la modification des droits de visite du père. L'enfant était né en Espagne, pays dans lequel a résidé continuellement jusqu'en février 2010, date à laquelle la mère, séparée avec le père, s'était établie au Royaume Uni. En juillet 2010, les parents ont arrivé à un accord sur la custodie de l'enfant, homologué par un juge espagnol devant lequel les deux se sont présentés volontairement. Peu de temps après, la mère s'était adressée aux instances anglaises de la nouvelle résidence de l'enfant sollicitant la modification du jugement espagnol ; leur compétence a été contestée, le père invoquant la prorogation de compétence intervenue en faveur des instances espagnoles. Suite à l'appel déclaré contre l'arrêt par lequel High Court s'est déclarée compétente, la Court of Appeal a adressé à la Cour de justice une série de questions préliminaires intéressant, entre autres, le problème de savoir si « lorsque la compétence d'une juridiction d'un État membre a été prorogée en vertu de l'article 12, paragraphe 3, du [règlement $n$ o 2201/2003] en ce qui concerne des questions de responsabilité parentale, cette prorogation produit-elle seulement ses effets jusqu'au prononcé d'une décision passée en force de chose jugée dans la procédure en question ou ces effets perdurent-ils? "

59 Par ailleurs, la même règle a été appliquée dans l'affaire par les juges espagnols aussi ; saisis par la mère en décembre avec une demande de transfert de la compétence vers les juridictions anglaises, sur le fondement de l'article 15 , ont rejeté la sollicitation avec l'argumentation suivante : " [d]ans la mesure où la [décision du 20 octobre 2010] rendue dans cette affaire [était] passée en force de chose jugée, où l'affaire [était] close et où aucune autre affaire opposant les parties en matière familiale ne demeur[ait] pendante devant le tribunal, il n'exist[ait] aucune raison de constater le défaut de compétence comme demandé dans la requête " (v. par. 22 de l'arrêt). V. aussi CJUE, 15 février 2017, C-499/15, W et $V$ contre $X$, par. 70 : " II résulte de l'ensemble des considérations qui précèdent qu'il convient de répondre à la question posée que l'article 8 du règlement $n^{\circ}$ 2201/2003 et l'article 3 du règlement $n^{\circ} 4 / 2009$ doivent être interprétés en ce sens que, dans une affaire telle que celle au principal, les juridictions de l'État membre qui ont adopté une décision passée en force de chose jugée en matière de responsabilité parentale et d'obligations alimentaires concernant un enfant mineur ne sont plus compétentes pour statuer sur une demande de modification des dispositions arrêtées par cette décision, dans la mesure où la résidence habituelle de l'enfant est située sur le territoire d'un autre État membre. Ce sont les juridictions de ce dernier État membre qui sont compétentes pour statuer sur cette demande ".

60 V. le par. 48 de l'arrêt : " l'article 12, paragraphe 3 , visant à permettre aux titulaires de la responsabilité parentale de saisir d'un commun accord et sous certaines autres conditions une juridiction de sujets relevant de la responsabilité parentale pour l'appréciation desquels elle n'est pas, en principe, compétente, il ne saurait être présumé qu'un tel accord persiste, dans tous les cas, au-delà du terme de la procédure engagée et en ce qui concerne d'autres sujets pouvant se présenter ultérieurement $»$. 
${ }^{61}$ V. E. Pataut, op. cit., p. $157, \mathrm{n}^{\circ} 53$.

${ }^{62}$ Dans la pratique roumaine, les instances ont décidé que l'accord n'existe pas lorsqu'une des parties - très souvent le défendeur, résidant en Roumanie (le demandeur et l'enfant ayant leur résidence à l'étranger) - se présente devant les juridictions pour contester la compétence (Trib. $1^{\text {re }}$ instance Bistrita, ch. civ., déc. $n^{\circ}$ 10314/2013, du 19 décembre 2013, confirmé par le Trib. Bistrita Nasaud, $1^{\text {re }}$ ch. civ., déc. $n^{\circ} 76 / A / 2014$, du 28 mai 2014 ; v. dans le même sens Trib. Buzau, $1^{\text {re }}$ ch. civ., déc. $n^{\circ} 57 / 2016$ du 21 mars 2016). Une solution identique est retenue pour le cas de la non comparution de défendeur - v. Trib. $1^{\text {re }}$ instance Moreni, déc. $n^{\circ} 311$ du 23 juin 2014 ; Trib. $1^{\text {re }}$ instance Bacau, ch. civ., déc. $n^{\circ} 2105$ du 2 avril 2015 ; v. également Trib. $1^{\text {re }}$ instance Moreni, déc. $n^{\circ} 1098$ du 17 décembre 2012 (décision dans laquelle, discutant l'exigence de l'accord, mentionnée à l'article 12.3.b) du règlement, la cour a précisé expressément que la prorogation conventionnelle de la compétence ne peut pas opérer suite à la manifestation de volonté exclusive d'une seule des parties (le demandeur) et a exigé que l'accord des parties sur la compétence survienne avant la saisine de l'instance.

63 Par exemple, dans la pratique roumaine, le Tribunal de première instance Tg. Mures a considéré qu'il n'a pas été correctement saisi sur la base de l'article 12.3 du règlement en raison de l'absence de déclaration expresse d'acceptation de la compétence par le défendeur (même si celui-ci, présent dans la procédure, s'est déclaré d'accord avec les prétentions du demandeur sur le fond); l'instance a décidé, également, que l'intérêt supérieur de l'enfant sera mieux respecté si le litige serait tranché par les instances belges de l'État de résidence habituelle de l'enfant, où pouvait être faite directement et plus adéquatement la probation sur le milieu de vie de l'enfant et ses relations sociales et familiales - v. Trib. $1^{\text {re }}$ instance Tg. Mures, ch. civ., déc. $n^{\circ} 3588$, du 30 juin 2016. Le Tribunal Mures a eu une position similaire dans une affaire tranchée également en 2016, concernant le divorce d'un couple des Roumains et la custodie de leurs enfants, toutes les parties impliquées ayant leur résidence habituelle en Italie : avec une motivation précaire à notre avis, centrée sur l'importance de la proximité, les juges se sont fondés exclusivement sur l'intérêt supérieur de l'enfant et ont refusé de retenir leur compétence, même si les parents, citoyens Roumains, se sont mis d'accord indirectement sur la compétence, en mettant des conclusions exclusivement sur le fond, tant dans la première instance, que dans l'instance de recours $-v$. Trib. Mures, ch. civ., déc. n 172 du 25 février 2016.

${ }^{64} \mathrm{Ce}$ " conflit » ne sera pas résolu sur la base de la règle de litispendance figurant à l'article $19 \mathrm{du}$ règlement. Au contraire, la hiérarchie établie entre les articles 8 et 12 et les critères de compétence correspondants jouera son rôle : si en général, le jeu de l'autonomie de la volonté est admissible en la matière, en l'absence de l'accord des parties, la compétence des instances de l'État de la résidence habituelle de l'enfant est prioritaire et doit être suivie, de sorte qu'en conformité avec I'article 17 toute autre juridiction doit se déclarer incompétente.

${ }^{65}$ V. dans le même sens E. Gallant, Note précitée, p. 679.

${ }^{66}$ CJUE, 21 octobre 2015, C-215/15, Gogova c. lliev, Rev. Trim. Dt. Eur, 2015, p. 803, note V. Egéa. Le litige impliquait les parents bulgares, non mariés, d'un enfant âgé de 10 ans. Même si tant les parents que l'enfant avaient leur résidence habituelle en Italie, la mère a commencé devant les juridictions bulgares une procédure dont l'objet était la supplantation du consentement du père pour la délivrance d'un passeport pour l'enfant. Se trouvant dans l'impossibilité de lui notifier la requête introductive d'instance, celles-ci ont désigné pour la représentation du père un mandataire 
ad litem ; en constatant ultérieurement que la résidence habituelle de l'enfant est en Italie, elles se sont déclarées néanmoins incompétentes. L'ordonnance correspondante a été attaquée, la mère soutenant que l'absence de la contestation de la compétence par le mandataire ad litem vaut accord pour la prorogation de la compétence.

${ }^{67}$ V. la question préliminaire reformulée par la Cour de justice : « il y a lieu de comprendre la troisième question comme visant à déterminer, en substance, si l'article 12, paragraphe 3 , sous b), du règlement $n^{\circ} 2201 / 2003$ doit être interprété en ce sens que la compétence des juridictions saisies pour connaître d'une demande en matière de responsabilité parentale peut être considérée comme ayant été «acceptée expressément ou de toute autre manière non équivoque par toutes les parties à la procédure", au sens de cette disposition, au seul motif que le mandataire ad litem représentant le défendeur, désigné d'office par ces juridictions au regard de l'impossibilité de notifier à ce dernier la requête introductive d'instance, n'a pas soulevé l'incompétence desdites juridictions » (par. 39).

${ }^{68}$ La même interprétation stricte est par ailleurs mise en évidence par l'arrêt $E$ (par. 48).

69 V. antérieurement CJCE, 10 octobre 1996, C-78/95, Hendrikman et Feyen, dans le contexte de l'interprétation de la Convention de Bruxelles de 1968: "un défendeur qui ignore la procédure entamée à son encontre et pour qui comparaît, devant le juge d'origine, un avocat qu'il n'a pas mandaté se trouve dans l'impossibilité absolue de se défendre " (par. 18). V. également CJUE, 11 septembre 2014, C-112/13, A c. B, arrêt relatif à l'interprétation de l'article 24 du règlement $44 / 2001$ et dans lequel la haute juridiction a décidé que la comparution dans la procédure du curateur du défendeur absent et de domicile inconnu ne peut pas justifier la prorogation (tacite) de la compétence : « la prorogation tacite de compétence en vertu de l'article 24 , première phrase, du règlement no 44/2001 est fondée sur un choix délibéré des parties au litige relatif à cette compétence, ce qui présuppose que le défendeur ait connaissance de la procédure entamée contre lui. En revanche, un défendeur absent auquel la requête introductive d'instance n'a pas été lui notifiée et qui ignore la procédure entamée contre lui ne peut être considéré comme acceptant tacitement la compétence du juge saisi. En outre, un défendeur absent ignorant le recours introduit contre lui ainsi que la nomination d'un curateur du défendeur absent ne peut fournir à ce curateur toutes les informations nécessaires pour apprécier la compétence internationale de la juridiction saisie et lui permettre de contester effectivement cette compétence ou de l'accepter en connaissance de cause. Dans ces circonstances, la comparution dudit curateur du défendeur absent ne saurait non plus être considérée comme valant acceptation tacite de ce défendeur " (par. 54-55 de l'arrêt, sur lequel v. P. Callé, Note, Rev. Crit. DIP, 2015, p. 915-921, spéc. p. 919-921, n 15-21).

70 La position libérale de ce texte est bien-connue : la prorogation tacite est efficace. II y a une acceptation tacite de la compétence lorsque le défendeur se présente dans la procédure et formule des conclusions sur le fond, sans contester la compétence : v. par exemple CJCE, 7 mars 1985, Hannelore Spitzley, Aff. 48/84; CJUE, 17 mars 2016, C-175/15, Taser international. En détail, v. H. Gaudemet-Tallon, Compétence et exécution des jugements en Europe, Matières civile et commerciale, 5e éd., LGDJ, 2015, p. 186 et s., n 163 et s.

${ }^{71}$ Cela se justifie d'autant plus que la compétence de la juridiction d'origine ne peut plus être vérifiée au stade de l'instance indirecte et que la matière intéresse le statut personnel, caractérisé par l'indisponibilité de principe des droits. 
72 Dans la pratique des instances roumaines, la prorogation tacite a été admise : v. par exemple Trib. lași, $1^{\text {re }}$ ch. civ., déc. $n^{\circ} 258$ du 8 juin 2016, dans laquelle l'instance a décidé que la présence du défendeur dans la procédure, à des séances différentes, sans contester la compétence, peut être interprétée comme une acceptation non équivoque de la compétence. V. aussi Trib. $1^{\text {re }}$ instance Brăila, ch. civ., déc. $n^{\circ} 59$ du 23 janvier 2013, appréciant que le défendeur a accepté de manière non équivoque la compétence des juridictions roumaines, l'avocat qu'il avait volontairement désigné mettant les conclusions directement sur le fond (sans contester la compétence).

${ }^{73}$ V. par exemple Th. Rauscher, op. cit., spéc. p. 40. Dans le sens que la position de défendeur qui a connu l'existence de la procédure et qui a choisi de ne pas comparaître pourra être interprétée comme une acceptation tacite de la compétence, v. V. Egéa, Note sous l'arrêt Gogova, Rev. Trim. Dt. Eur, 2015, p. 805.

${ }^{74}$ Cette présence devrait toutefois démontrer non équivoquement l'acceptation de la compétence, les indices contraires attirant l'inapplicabilité de l'article 12 (dont les exigences doivent être strictement interprétées) ; ainsi, par exemple, dans la pratique roumaine, la Cour d'appel Galati a analysé l'exigence de l'accord prévue par ce texte et a décidé que la simple présence de demandeur à deux séances de jugement, sollicitant un ajournement de l'affaire afin de qu'il puisse s'engager un avocat, ne peut pas être interprétée comme une acceptation non équivoque de la compétence - C. Ap. Galati, déc. civ. n 106/R du 5 mars 2014.

${ }^{75}$ Sur cette solution, v. A.-L. Calvo Caravaca, J. Carascosa Gonzalez, "Article 26 », in U. Magnus, P. Mankowski (eds.), Bruxelles I bis Regulation, Otto Schmidt, 2016, p. 681-682, $n^{\circ}$ 33-35.

${ }^{76}$ V. B. Ancel, H. Muir-Watt, « L'intérêt supérieur... », op. cit., p. 587, $n^{\circ} 17$.

77 L'accord de prorogation permet d'éviter ou de limiter les dissensions entre les parents, ainsi que l'intérêt de l'enfant est lui-aussi soutenu. Par exemple, dans l'affaire $E$, l'acceptation de la compétence par les juridictions espagnoles devant lesquelles les deux parents se sont présentés volontairement malgré le fait que la mère était établie depuis une année avec l'enfant au Royaume Uni, a eu des effets bénéfiques sur la situation de l'enfant: l'intervention du juge pour l'homologation de leur entente a permis l'extinction, du moins partielle, de leur dispute, sans l'affectation négative de l'enfant du point de vue émotionnel et psychologique.

${ }^{78} \mathrm{~V}$. par ailleurs en ce sens la position de l'Avocat général P. Mengozzi, dans ses conclusions sous l'affaire Gogova, qui mentionne l'obligation de l'instance de s'assurer que les parties n'ont pas exercé en défaveur des intérêts de l'enfant l'autonomie qui leur est reconnue (v. par. 66 de l'Opinion présentée le 10 septembre 2015). V. aussi E. Pataut, op. cit., p. 153, n 37, soulignant toutefois le caractère plutôt exceptionnel des hypothèses de censure de l'accord des titulaires de la responsabilité parentale.

${ }^{79} \mathrm{~V}$. les arrêts $E$ (par. 47) et $L$ (par. 58)

80 V. par exemple P. Hammje, "L'intérêt de l'enfant face aux sources internationales du droit international privé ", in Le droit international privé : esprit et méthodes. Mélanges en l'honneur de Paul Lagarde, Dalloz, 2005, p. 365 et s.

${ }^{81}$ V. P. Hammje, op. cit., p. 372 , qui insiste sur la proximité.

82 L'exigence du lien étroit apparaît expressément dans l'article 12.3 et seulement indirectement (et plus atténuée, en raison de la multiplication des critères de compétence juridictionnelle prévus en matière de divorce) dans l'article 12.1 . 
${ }^{83}$ Dans sa jurisprudence récente, la Cour européenne de justice a montré toute son ouverture pour cet effet utile : par exemple, dans l'arrêt $W, V c$. $X$ du 17 février 2017 (C-499/15), elle a réaffirmé le caractère principiel de la compétence des juridictions de l'Etat de la résidence habituelle de l'enfant, établie bien entendu en tenant compte de l'intérêt supérieur de l'enfant (par. 60 et 64), mais rappelle aussi que les dérogations sont possibles en présence des éléments de fait particuliers : parmi ceux-ci, la Cour mentionne expressément un éventuel accord des titulaires de la responsabilité parentale sur la compétence des instances d'un autre Etat membre que celui de la résidence habituelle de l'enfant (par. 65).

${ }^{84}$ CJUE, 27 octobre 2016, aff. 428/15, Child and Family agency c. JD.

${ }^{85}$ Les conclusions de l'Avocat général M. Wathelet, présentées le 16 juin 2016, C-428/15, Child and Family Agency.

${ }^{86}$ V. en ce sens, dans la jurisprudence roumaine C. Ap. Galați, déc. n 106/R du 5 mars 2014, dans laquelle l'instance a apprécié que la prorogation de la compétence ne servirait pas l'intérêt supérieur de l'enfant car la probation relative à son milieu de vie et à ses relations familiales et sociales pourrait être faite plus facilement et plus adéquatement en Italie (pays dans lequel l'enfant résidait depuis 2007); l'argument du non-respect de l'intérêt supérieur de l'enfant a été toutefois invoqué non pour censurer un accord ferme des parents sur la prorogation, mais pour conforter la solution de rejet de la prorogation, dans le contexte du caractère discutable de l'acceptation de celle-ci par le défendeur. V. aussi Trib. Iași, $1^{\text {re }}$ ch. civ., déc. $n^{\circ} 258$ du 8 juin 2016 - dans cette affaire concernant une famille de Roumains (les enfants résidant à l'étranger avec la mère, demandeur dans la procédure), le tribunal a argumenté le respect de l'intérêt supérieur de l'enfant par le fait que les audiences allaient se dérouler en Roumain, langue commune des parties, sans nécessiter des dépenses supplémentaires de communication et de traduction des actes de procédure, ainsi que par le fait que la probation nécessaire à la solution de la dispute avait été déjà administrée devant les instances de fond. 